ARTICLE

\title{
Docking of acetyl-CoA carboxylase to the plastid envelope membrane attenuates fatty acid production in plants
}

Yajin Ye ${ }^{1}$, Krisztina Nikovics ${ }^{2}$, Alexandra To (10 ${ }^{2}$, Loïc Lepiniec (10 ${ }^{2}$, Eric T. Fedosejevs ${ }^{1}$, Steven R. Van Doren (1) ${ }^{1}$, Sébastien Baud (10 ${ }^{2} \&$ Jay J. Thelen (10 ${ }^{1 \times}$

In plants, light-dependent activation of de novo fatty acid synthesis (FAS) is partially mediated by acetyl-CoA carboxylase (ACCase), the first committed step for this pathway. However, it is not fully understood how plants control light-dependent FAS regulation to meet the cellular demand for acyl chains. We report here the identification of a gene family encoding for three small plastidial proteins of the envelope membrane that interact with the $\alpha$-carboxyltransferase $(\alpha-C T)$ subunit of ACCase and participate in an original mechanism restraining FAS in the light. Light enhances the interaction between carboxyltransferase interactors (CTIs) and $\alpha-\mathrm{CT}$, which in turn attenuates carbon flux into FAS. Knockouts for CTI exhibit higher rates of FAS and marked increase in absolute triacylglycerol levels in leaves, more than 4-fold higher than in wild-type plants. Furthermore, WRINKLED1, a master transcriptional regulator of FAS, positively regulates CTI1 expression by direct binding to its promoter. This study reveals that in addition to light-dependent activation, "envelope docking" of ACCase permits fine-tuning of fatty acid supply during the plant life cycle.

\footnotetext{
${ }^{1}$ Department of Biochemistry, Christopher S. Bond Life Sciences Center, University of Missouri-Columbia, 1201 E Rollins, Columbia, MO 65211, USA. ${ }^{2}$ Institut

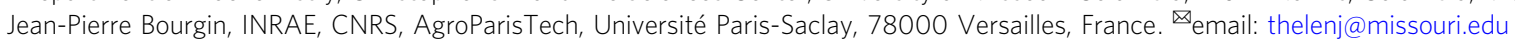


$\mathrm{V}$ egetable oils are an important source of food, renewable energy, and industrial feedstocks. As demand for this commodity steadily increases, development of improved oil crops has become an agronomic priority ${ }^{1}$. Oil biosynthesis begins with the de novo fatty acid synthesis (FAS) pathway in the plastids. The acyl chains thus obtained are eventually esterified to glycerol to produce triacylglycerols (TAGs), the major form of storage lipid in plants $^{1}$. Like many biosynthetic processes in plastids, the overall rate of FAS oscillates with light/dark cycles, increasing in the light and decreasing in the dark. Upon light illumination, stromal $\mathrm{pH}, \mathrm{Mg}^{2+}, \mathrm{ATP} / \mathrm{ADP}$ ratio, and redox potential increase in the chloroplasts, promoting carbon fixation and $\mathrm{FAS}^{2,3}$. Light-dependent control of FAS is partially achieved by regulation of acetyl-coenzyme A carboxylase (ACCase; EC.6.4.1.2) which catalyzes the first committed step in FAS, the ATP-dependent formation of malonyl-CoA from acetyl-CoA and bicarbonate $e^{4,5}$. In the plastids of dicots and non-graminaceous monocots, the predominant form of ACCase is a multisubunit, heteromeric complex (htACCase) comprised of four distinct subunits named biotin carboxylase (BC), biotin carboxyl carrier protein (BCCP), and $\alpha$ - and $\beta$-carboxyltransferases (CTs). In vitro, increasing $\mathrm{pH}, \mathrm{Mg}^{2+}, \mathrm{ATP} / \mathrm{ADP}$ ratio, as well as reducing conditions activates ACCase ${ }^{4,5}$. Additionally, ACCase is subject to feedback inhibition by downstream metabolites ${ }^{6,7}$ and effector proteins like the PII protein, a signal integrator that interacts with BCCP subunits to reduce the Vmax of the enzyme ${ }^{8}$. Additionally, a set of noncatalytic biotin/lipoyl attachment domain-containing (BADC) proteins are involved in the regulation of ACCase activity, including light-dark modulation ${ }^{9-11}$.

Here, we report the isolation and characterization of a gene family, CARBOXYLTRANSFERASE INTERACTORs (CTIs), encoding three plastid membrane proteins, which are involved in the regulation of de novo FAS. Using the noncatalytic domain of a-CT as bait in a yeast two-hybrid screen, we identified CTI1, an uncharacterized gene. The cti mutants display higher rates of FAS and higher TAG content in leaves. Expression of CTI1 is activated by the WRINKLED1 transcription factor. Aside from its overall activating role in FAS, light can simultaneously attenuate FAS by promoting interactions between $\alpha$-CT and CTIs, which in-turn inhibit htACCase activity and subsequently fatty acid synthesis. Our results provide important insights into how plants control light-dependent FAS to meet the cellular demand for acyl chains.

\section{Results}

The noncatalytic domain of a-CT directly interacts with CTIs. In seed plants, the $\alpha$-CT subunit of htACCase contains a large, noncatalytic C-terminus predicted to have coiled-coil structure, a structural motif that typically mediates protein-protein interactions ${ }^{12}$. This observation prompted us to identify potential interactors with the putative coiled-coil domain of a-CT. Using the C-terminus (amino acid 420-769) of Arabidopsis thaliana a$\mathrm{CT}$ as bait, a yeast two-hybrid (Y2H) screen was performed. Among 16 candidates, the screen identified the C-terminus of At1g42960 from two different clones. This candidate was named CARBOXYLTRANSFERASE INTERACTOR1 (CTI1). The sequence of the CTI1 protein contains a chloroplast transit peptide, a single transmembrane domain, followed by a coiledcoil domain (Supplementary Fig. 1a-d and Supplementary Table 1). By surveying the Arabidopsis genome, we found two CTI1 homologs, CTI2 (At3g02900) and CTI3 (At5g16660), and the three CTIs share similar predicted protein domains (Supplementary Fig. 1a-d and Supplementary Table 1). Based on phylogenetic analysis (Supplementary Fig. 2, and Supplementary Table 2), the CTI family appears to be of cyanobacterial origin, with the C-terminal domain conserved between plants and cyanobacteria. While green algae, bryophytes, and gymnosperms generally possess a single CTI, an apparent duplication event in an ancestral angiosperm gave rise to two divergent angiosperm CTI subfamilies, one of which includes Arabidopsis CTI1, while the other includes the closely-related Arabidopsis CTI2/3 genes. Interestingly, the CTI1 subfamily is apparently absent from grasses that lack the heteromeric form of ACCase. In contrast, the CTI2/3 subfamily is conserved across major angiosperm groups.

To confirm the interaction between $a-C T$ and CTI1, we cloned the coiled-coil-containing C-terminus of CTI1 (Supplementary Fig. 1a) and full-length $\alpha$-CT for targeted $\mathrm{Y} 2 \mathrm{H}$ assays. The results confirmed an interaction of $\alpha$-CT with CTI1, as well as with the CTI2 and 3 proteins (Fig. 1a). To control for assay specificity, the interactions between CTIs and $\beta$-CT, TRIGALACTOSYLDIACYLGLYCEROL2 (TGD2) ${ }^{13}$ or the coil-coiled-containing PROTEIN TARGETING TO STARCH2 (PTST2) ${ }^{14}$ with a-CT were tested; the results showed no interactions between either of these pairs (Fig. 1a). Next, we conducted bimolecular fluorescence complementation (BiFC) assays using a split YFP experimental system in Arabidopsis protoplasts. When CTI1-nYFP and $a-C T$ cYFP were coexpressed in protoplasts, a YFP signal was detectable that colocalized with chlorophyll, confirming a-CT/CTI1 interaction in planta (Fig. 1b). In contrast, no interaction could be detected between CTI1 and BCCP2 or PTST2. Additionally, both CTI2 and 3 interacted with $\alpha-C T$ in BiFC assays (Supplementary Fig. 3a, b). Binding affinity of the CTIs for $a-C T$ was then quantified by microscale thermophoresis (MST) using the Cterminal sequence of both CTIs and $a-C T$. CTI1 exhibited a 10and 100-fold higher affinity for a-CT compared to CTI3 and 2, respectively, based upon $K_{D}$ values (Fig. 1c). We thus identified a family of proteins that associate with htACCase through direct interaction with $a-C T$.

CTI proteins are localized in the chloroplast inner envelope membrane. Consistent with in silico predictions, CTIs were previously detected in chloroplast envelope membranes ${ }^{15}$. Transient expression of constructs encoding for CTI-YFP fusion proteins in Arabidopsis protoplasts revealed discrete foci at the periphery of chloroplasts similar to that observed with a-CT-YFP (Fig. 2a) and TGD2-GFP fusions ${ }^{13}$. This pattern of localization also mirrored the distribution of two other ACCase interactors, i.e., PII and BADC1 proteins, in plastids ${ }^{10,16}$. It is not clear, however, whether or not these foci denote subdomains of the membrane where htACCase units are preferentially clustered in a wild-type context, considering that protein overexpression produced similar patterns ${ }^{17}$. To specifically confirm colocalization of CTI1 with TGD2, a well-characterized protein of the plastid inner envelope membrane ${ }^{13}$, or with the CT subcomplex of htACCase previously shown to be associated with the plastid inner envelope membrane ${ }^{18-20}, C T I 1-G F P$ was transiently coexpressed with $\alpha$ CT-RFP (Fig. 2b) or with TGD2-RFP (Fig. 2c) in leaves of Nicotiana benthamiana. Colocalization of GFP and RFP signals suggested that CTI1 is indeed a component of the chloroplast inner envelope membrane. Furthermore, co-immunolocalization experiments were carried out between CTI1 and E37, a methyltransferase involved in tocopherol and plastoquinone synthesis localized to the plastid inner envelope membrane ${ }^{21-23}$. In Arabidopsis protoplasts transfected with the Pro35S:CTI1:HA construct and in embryonic cells of Arabidopsis transgenic lines stably transformed with the ProCTI1:CTI1:HA construct, the CTI1:HA fusion protein appeared as a punctate pattern colocalized with a subset of the wider distribution of E37, confirming targeting of CTI1 to the chloroplast inner envelope membrane (Fig. 2d). Finally, the membrane topology of CTI1 was determined by dual-protease digestion assay. Intact chloroplasts were 


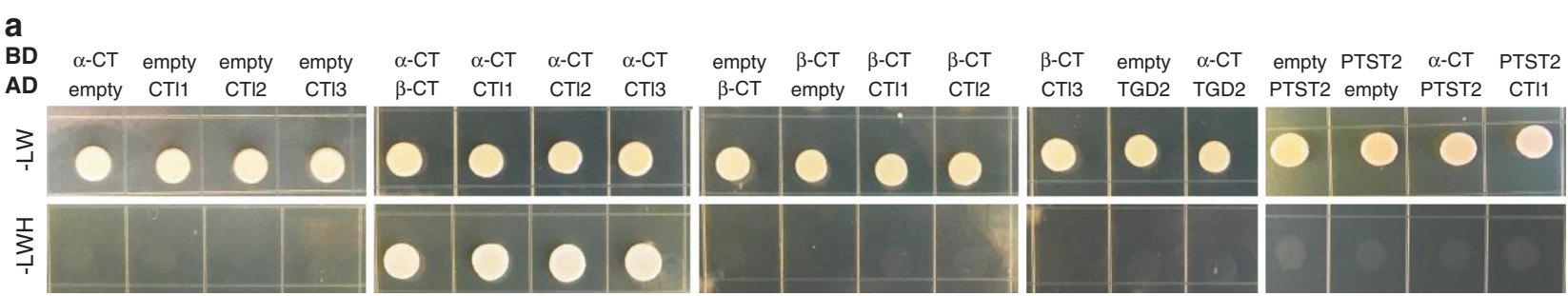

b

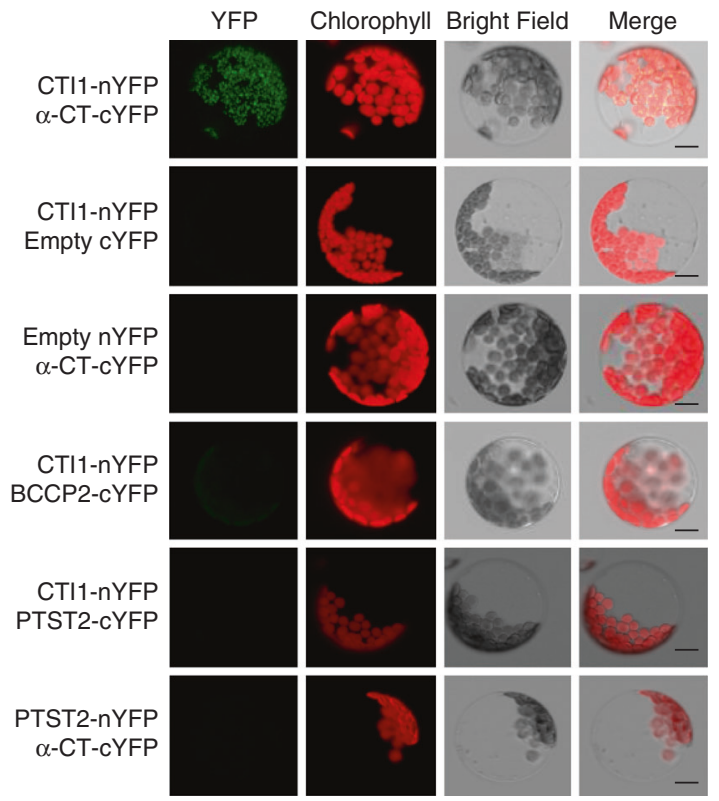

C

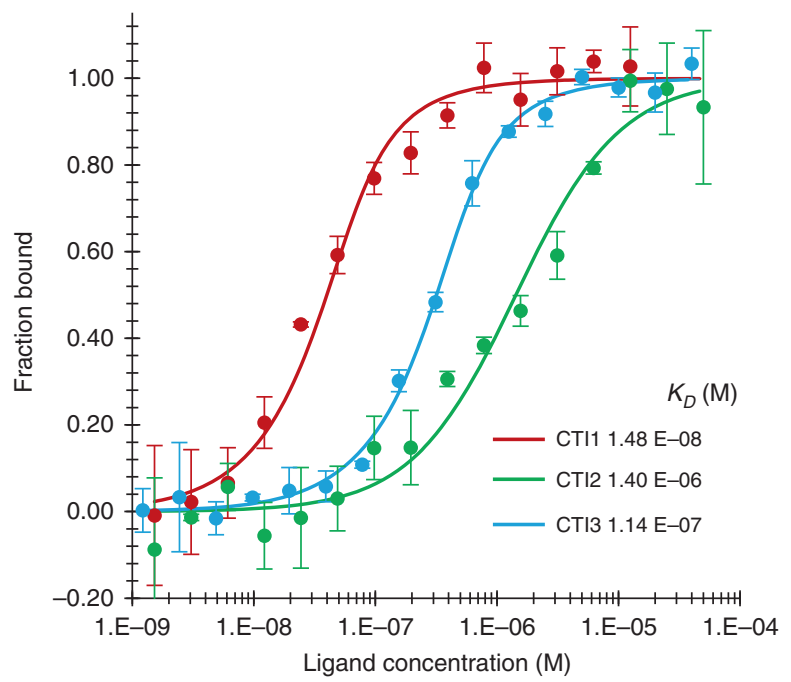

Fig. 1 CTIs physically interact with $\boldsymbol{\alpha}$-CT in vitro and in vivo. a Yeast two-hybrid assay testing interactions between pairs of proteins. Each protein tested was fused to a GAL4 DNA-binding domain (BD) or to a GAL4 activation domain (AD). Representative cells grown on synthetic dropout (SD)-LW or SDLWH medium with $1 \mathrm{mM}$ 3-amino-1,2,4-triazole are presented. The experiment was repeated three times with similar results. $\mathbf{b}$ BiFC assays. Experiments were repeated three times with similar results. For each of the three independent protoplast transformations, at least three different protoplasts were observed. Bars $=10 \mu \mathrm{m}$. c Microscale thermophoresis (MST). Data points indicate the fraction of labeled $\alpha$-CT coiled-coil domain bound to CTIs. Dissociation constants $\left(K_{D}\right)$, expressed in $M$, are presented. Data points are means $\pm S D, n=3$ individual biological replicates.

isolated from Arabidopsis leaf tissues and treated with thermolysin (a protease unable to penetrate the outer envelope membrane) or trypsin (a protease able to penetrate the outer envelope but not the inner envelope membrane). CTI1, like $\alpha$-CT, was resistant to both proteases, suggesting that the C-terminus of the CTI1 protein faces the stromal side of the inner envelope membrane (Fig. 2e).

CTIs negatively regulate fatty acid synthesis through htACCase. To determine the function of CTIs in Arabidopsis, we used CRISPR/Cas9 to knockout the CTI genes individually. We recovered CRISPR/Cas9-induced homozygous frameshift mutants for each CTI gene (Supplementary Fig. 4a). The frameshifts induced premature termination codons preceding the coiled-coil domain for all three CTIs (Supplementary Fig. 4b). The efficacy of the ctil knockout mutant was confirmed by immunoblotting using anti-CTI1 antibody (Supplementary Fig. 4c). Double and triple mutants were then generated by crosses to examine genetic interactions among the CTIs. When grown under long-day conditions, the cti123 triple mutant displayed a growth defect with smaller rosette leaves and decreased aerial biomass compared to single and double mutants or wild-type (WT) plants, indicating a growth penalty in the triple mutant (Fig. 3a, b).

We then assayed ACCase activity in leaves of the different genotypes. Soluble protein fractions from crude chloroplasts prepared from wild-type leaves were assayed in the presence of two different ACCase inhibitors; avidin, a general inhibitor of
ACCase activity, and the herbicide haloxyfop, which specifically targets homomeric ACCase ${ }^{24}$. Whereas addition of avidin strongly reduced the measured ACCase activity, haloxyfop did not cause a significant reduction in specific activity (Supplementary Fig. 5), demonstrating that htACCase activity predominates in the plant material prepared. Then, supernatants and pellets from clarified $(13,000 \times g)$ chloroplast lysates from 4-week-old leaves of the different cti mutants were assayed for soluble and membranebound ACCase activity, respectively. In the supernatants, the ctil mutation yielded a 3.5-fold increase in ACCase activity with respect to the WT control. ACCase activity was further enhanced in cti13 and cti123 mutants (Fig. 3c). Conversely, in the pellets, the cti1 mutation yielded lower ACCase activity, and further reduction was observed in the cti123 triple mutant (Fig. 3c). To estimate the rate of FAS in cti mutants, we performed in vivo ${ }^{14} \mathrm{C}$-acetate labeling experiments using 4-week-old leaf strips. After an incubation of $40 \mathrm{~min}$, the rate of ${ }^{14} \mathrm{C}$-acetate incorporation was around 3.5-fold higher in ctil than in WT, while a slight increase was observed in cti3 mutant. The double and triple mutants exhibited enhanced rates of incorporation with respect to the single mutants (Fig. 3d), reflecting the variations observed in soluble ACCase activity (Fig. 3c). Incorporation of ${ }^{14} \mathrm{C}$-malonate into nascent fatty acids was not higher in cti mutants (Supplementary Fig. 6). Next, we quantified htACCase subunit levels in the cti mutants by western blotting. The results indicated that higher FAS rates were not the consequence of increased levels of htACCase subunits (Supplementary Fig. 7). 
a
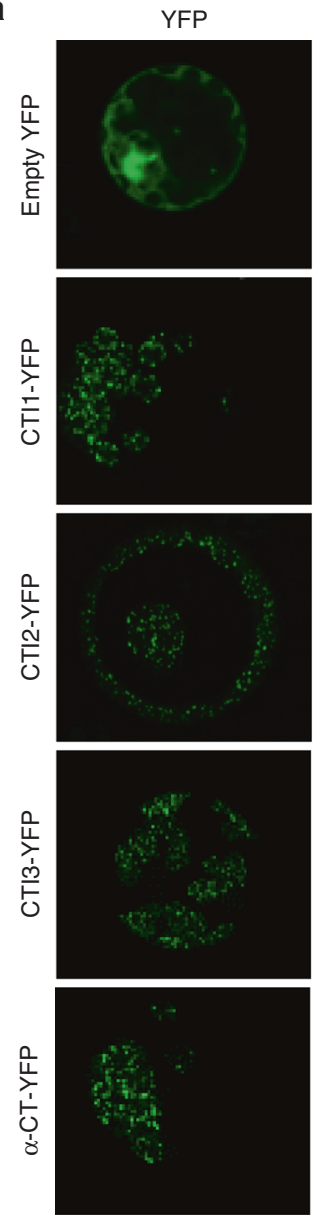

d

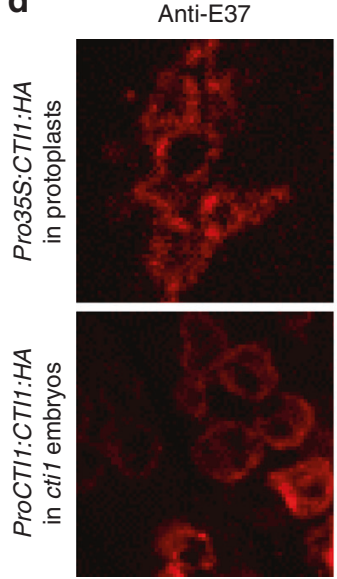

Chlorophyll
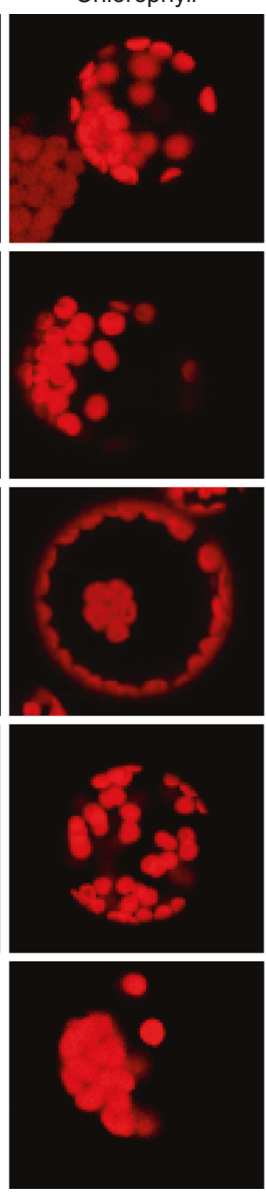

Anti-HA
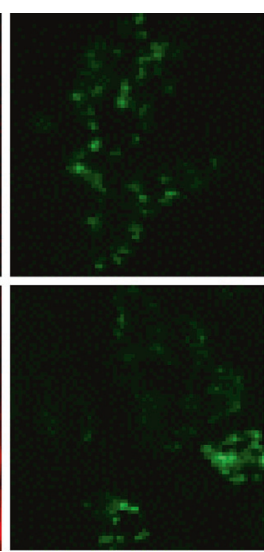
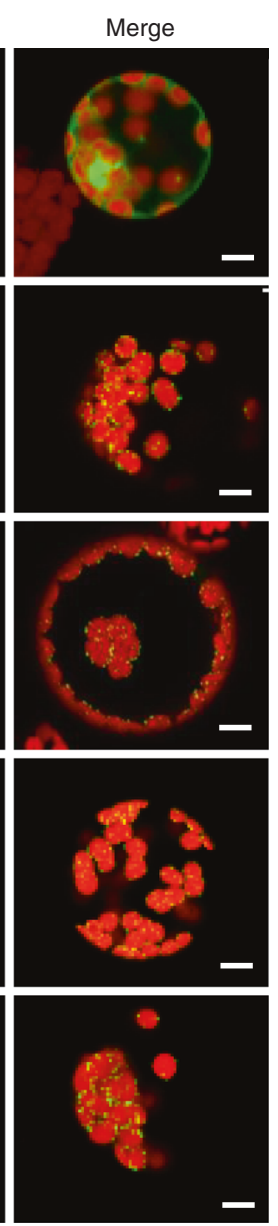

$-$

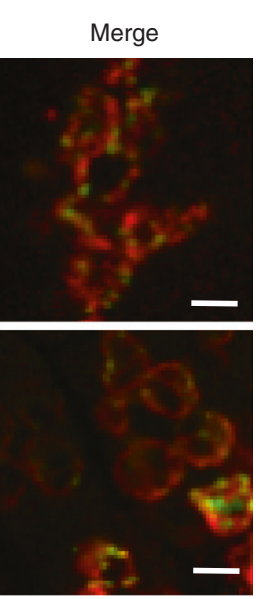

b

CTI1-GFP

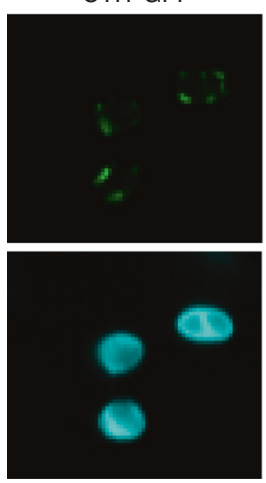

Chlorophyll

C

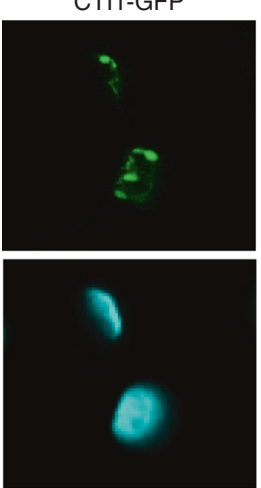

Chlorophyll
$\alpha$-CT-RFP
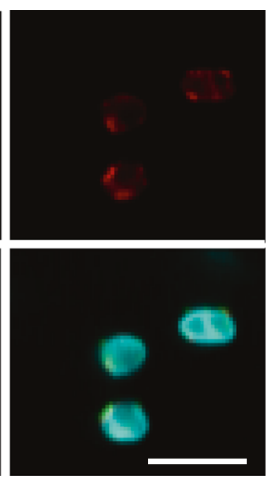

Merge

TGD2-RFP

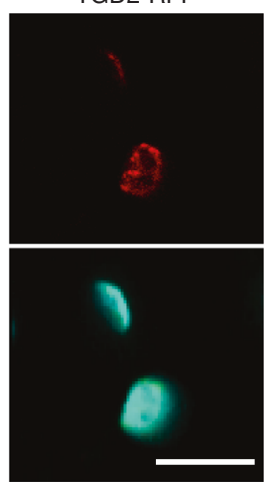

Merge

e

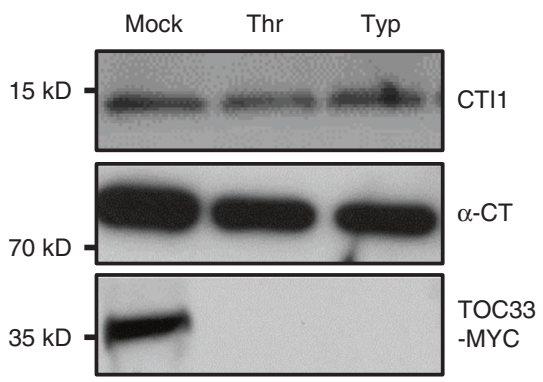

As a first approach to study the impact of higher soluble htACCase activity in cti mutants, we determined the total fatty acid content in leaves of plants grown under long-day (LD) photoperiod. Consistent with increased FAS rate, leaf fatty acid content in the ctil mutant was significantly higher than WT (a $22 \%$ increase). An additive effect of the cti mutations could be observed in double and triple mutants (Fig. 3e). The cti mutants also showed altered leaf fatty composition, with a decrease in the relative proportion of $18: 3$ polyunsaturated fatty acids and a concomitant increase in 18:0, 18:1, and 18:2 yielding a reduced desaturation ratio (Fig. 3f). Analysis of leaf lipid extracts by thinlayer chromatography (TLC) revealed accumulation of neutral lipids comigrating with TAGs in cti mutants (Fig. 3g).
Comprehensive, quantitative lipidomic analyses confirmed specific accumulation of TAGs in ctil and cti3 single mutants as well as an additive effect in double mutants (Fig. $3 \mathrm{~h}$ ). In contrast, TLC and quantitative lipidomic analyses of polar lipids showed that these classes of lipids were not affected by the cti mutations (Supplementary Fig. 8a, b). In mutant seeds, the total seed oil content was unmodified but a reduced desaturation ratio was observed as in leaves (Supplementary Fig. 9). To confirm the CRISPR mutant phenotypes, we then characterized a T-DNA insertion mutant corresponding to a second null allele of CTII (Supplementary Fig. 10a). This T-DNA allele (cti1-2) phenocopied the ctil CRISPR/Cas9 allele, including increased soluble ACCase activity, FAS rate, total fatty acid content, and TAG 
Fig. 2 CTIs are localized in the inner envelope membrane of chloroplasts. a Localization of CTIs and $\alpha$-CT in Arabidopsis protoplasts. CTIs and $\alpha$-CT were fused with YFP, and expressed in protoplasts. The fluorescence was detected by confocal microscopy $16 \mathrm{~h}$ after transformation. Empty vector was used as a control. Experiments were repeated three times with similar results. For each of the three independent protoplast transformations, at least five different protoplasts were observed. Bars $=5 \mu \mathrm{m}$. b Colocalization of CTI1-GFP and $\alpha$-CT-RFP fusions transiently coexpressed in leaves of Nicotiana benthamiana and observed two days after infiltration. Experiments were repeated three times with similar results. For each of the three independent transformations, at least five different cells were observed. Bars $=5 \mu \mathrm{m}$. c Colocalization of CTI1-GFP and TGD2-RFP fusions coexpressed in leaves of Nicotiana benthamiana and observed two days after infiltration. Experiments were repeated three times with similar results. For each of the three independent transformations, at least five different cells were observed. Bars $=5 \mu \mathrm{m}$. d Co-immunolocalization of CTI1 and the E37 marker of the inner envelope membrane of chloroplasts. Coimmunolocalization experiments were carried out in using anti-E37 primary antibodies and secondary antibodies conjugated to Alexa Fluor 568 (fluorescence observed at 603-682 nm; red signal) or specific anti-HA antibodies and secondary antibodies conjugated to Alexa Fluor 488 (fluorescence observed at 495-585 nm; green signal). Merged pictures are also presented. Experiments with protoplasts were repeated three times with similar results. For each of the three independent protoplast transformations, eight different protoplasts were observed. Experiments with embryos were repeated twice with similar results. In each experiment, 10 different cells from distinct embryos were observed. Bars $=5 \mu \mathrm{m}$. e Topology of the CTI1 protein. Intact Arabidopsis chloroplasts were digested by thermolysin (Thr) or trypsin (Typ) for 30 min. Proteins were detected with anti-CTI1 or anti- $\alpha$-CT antibodies. TOC33-MYC fusion protein, used as a control, was detected with anti-MYC antibodies. The results shown are representative of three biologically independent samples.

accumulation in leaves (Supplementary Fig. 10b-f), as well as modified fatty acid composition of leaves and mature dry seeds (Supplementary Fig. 10g). This last phenotype was complemented by introgression of the ProCTI1:CTI1:HA or ProAT2S2:CTI1 construct in the ctil-2 mutant background (Supplementary Fig. 10g). A partial complementation of the ctil-2 seed oil phenotype could also be obtained by introgression of CTI2 and CTI3 cDNAs placed under the control of the seed-specific ProAT2S2 promoter ${ }^{25}$, but the efficiency of these phenotypic reversions was lower (Supplementary Fig. 10g). Taken together, these results demonstrate that CTIs attenuate soluble htACCase activity, so that cti mutations enhance carbon flux into fatty acid synthesis, resulting in TAG over accumulation in leaves. If the three CTIs have partially redundant functions, CTI1 appears to be the predominant effector of htACCase activity. The phenotypic impact of the CTI1 isoform is consistent with both higher affinity for $a-C T$ and phylogeny data showing that CTI1 coevolved with htACCase.

WRINKLED1 activates CTI1 expression through direct binding to CTI1 promoter. Expression patterns of the CTI genes were investigated using qRT-PCR (Supplementary Fig. 11a) and translational ProCTI:CTI-uidA fusions assayed in transgenic plants (Supplementary Fig. 11b-d). The three CTIs appeared to be ubiquitously expressed, and CTI1 mRNAs were more abundant than CTI2 or CTI3 mRNAs in all tissues analyzed, providing another possible explanation for the predominant regulatory role of CTI1. Furthermore, a peak of CTI1 transcript accumulation was detected at the onset of seed maturation and this pattern was not observed for CTI2 and CTI3. The expression pattern of CTI1 in seeds was similar to the profiles observed for several genes encoding FAS enzymes known to be transcriptionally activated by the WRINKLED1 (WRI1) transcription factor, which binds AW cis-regulatory elements within its target promoters ${ }^{25-28}$. Interestingly, an AW box could be identified in the CTI1 promoter but not in CTI2 and CTI3 promoters (Fig. 4a). The interaction between WRI1 and the CTI1 promoter was demonstrated using a yeast one-hybrid approach: the expression of WRI1-AD in the strain containing the HIS3 reporter gene under the control of the CTI1 promoter resulted in the specific growth of the strain on medium lacking histidine (Fig. 4b). To test the ability of WRI1 to directly activate the CTI1 promoter in planta, a ProCTI1:uidA reporter construct was then used in transactivation assays in $N$. benthamiana leaves (Fig. 4c). WRI1 was able to activate reporter constructs comprising CTI1 or BCCP2 promoters (positive control), showing a strong increase in $\beta$-glucuronidase (GUS) activity, but not the negative control 2-OXOGLUTARATE-
DEPENDENT DIOXYGENASE (ODD) promoter $^{29}$, which could be activated by MYB118 (Fig. 4c). To further evaluate the impact of WRI1 on the pattern of CTI1 promoter activity, the ProCTI1: uidA construct was stably expressed in transgenic Arabidopsis lines. Unlike ProCTI1:CTI1:uidA transgenic lines, ProCTI1:uidA lines displayed GUS staining in reproductive organs but not in vegetative parts of the plants (Supplementary Fig. 10, 11), indicating that important regulatory elements exist downstream of the translational start codon of CTI1. The construct was then introduced into Pro35Sdual:WRI1 lines by crosses, and a strong induction of ProCTI1 activity was detected in rosette leaves (Fig. 4d). In contrast to the observations made in the WT, ProCTI1 activity could not be detected in wril embryos (Fig. 4e). In agreement with these observations, CTI1 mRNA levels were significantly decreased in wril embryos, whereas higher CTI1 mRNA levels were measured in leaves of WRI1 overexpressors (Fig. 4f). Together, these results showed that WRI1 enhances the activity of the CTI1 promoter, in a similar way to the activities of promoters of genes coding core FA biosynthetic enzymes and regulators of htACCase like the BADC subunits and PII ${ }^{16,30}$.

CTI/a-CT module regulates fatty acid synthesis in a lightdependent manner. It was proposed that light-dependent ${ }^{14} \mathrm{C}$ acetate incorporation into fatty acids is due to the regulation of htACCase ${ }^{31-33}$. To determine if the CTIs are involved, rates of FAS in 4-week-old leaves were monitored using ${ }^{14} \mathrm{C}$-acetate labeling. Under a classical LD regime, incorporation rates were higher at the end of the day than at the end of the dark period for most genotypes. Increased ${ }^{14} \mathrm{C}$-acetate incorporation observed in cti mutants was moderated at the end of the dark period (Fig. 5a). The enhancement of ${ }^{14} \mathrm{C}$-acetate incorporation measured in $c t i$ mutants was abolished by an extended dark period $(24 \mathrm{~h})$ but recovered after a 16-h-light period (Fig. 5b). To exclude the possibility that the variations in incorporation rates measured were reflecting differences in unlabeled acetate in leaves, a competition assay with unlabeled acetate was performed. Before adding ${ }^{14} \mathrm{C}$-acetate, leaf tissues were incubated in the presence of unlabeled acetate for $10 \mathrm{~min}$. No competition effect could be detected in the WT nor in the ctil mutant (Supplementary Fig. 13). However, considering that acetate is not the principal carbon source for FAS in planta ${ }^{34,35}$, we next quantified the total leaf fatty acid content from Arabidopsis plants grown under various photoperiods. No significant modifications of the leaf fatty acid content in the cti mutants could be observed under short-day conditions. In contrast, increased fatty acid content was measured in cti leaves under long-day conditions and under constant light (Fig. 5c). Like the ${ }^{14} \mathrm{C}$-acetate labeling assay, fatty 
a

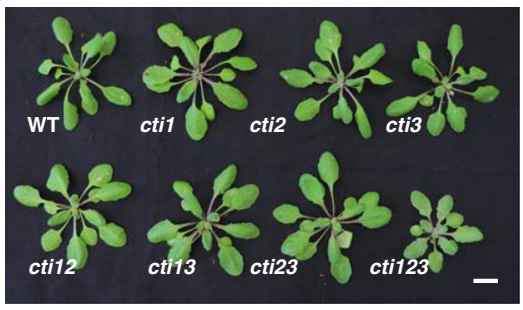

C
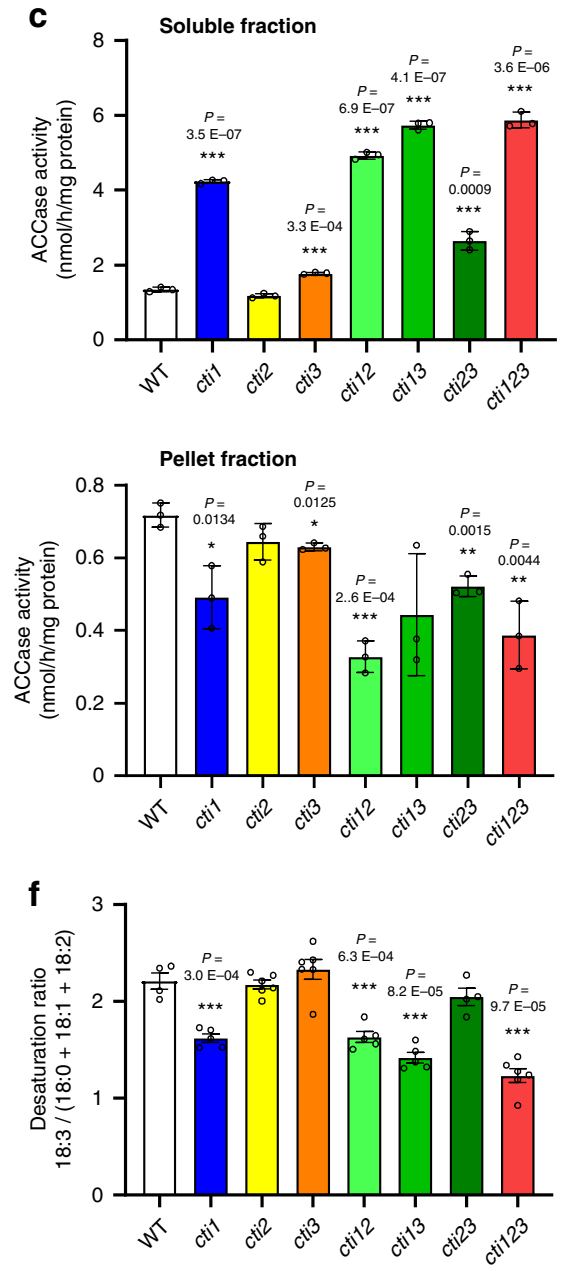

h

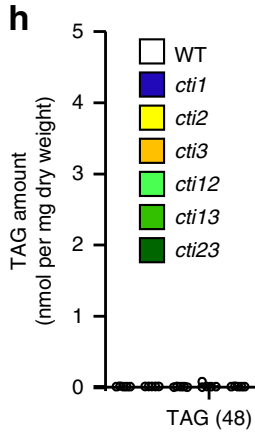

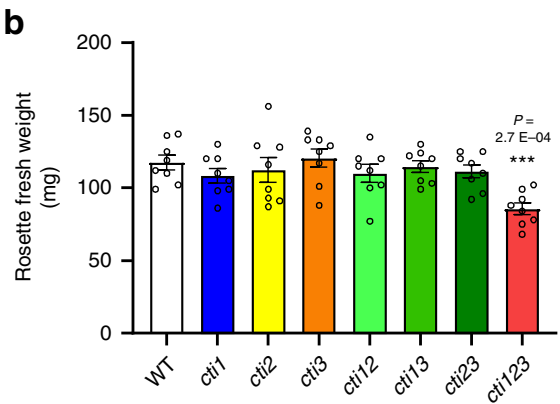

d

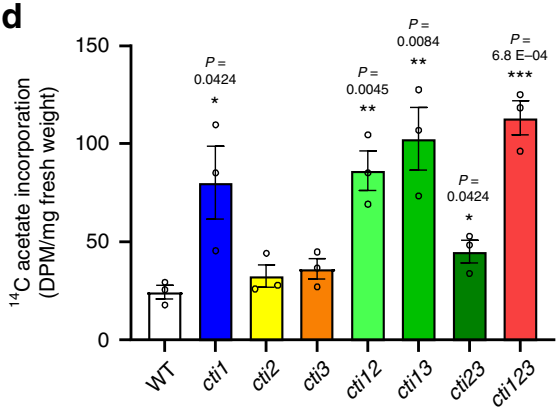

e

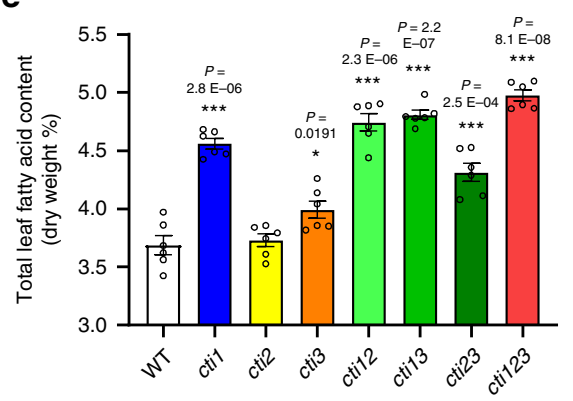

g

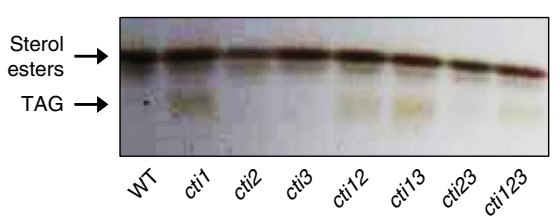

acid quantification suggested that CTIs inhibit FAS in the light. We finally tested whether a light-regulated CTI/a-CT interaction was responsible for the modulation of FAS. A split-luciferase assay was implemented in leaves of $N$. benthamiana. In leaves of plants grown $4 \mathrm{~d}$ under continuous light after agroinfiltration, a strong split-luciferase (LUC) signal could be detected (Fig. 5d). When agroinfiltrated leaves were kept $2 \mathrm{~d}$ in the light then $2 \mathrm{~d}$ in the dark, interactions between $\alpha$-CT and the CTIs appeared significantly weaker. However, a further $24 \mathrm{~h}$ recovery period in the light was sufficient to restore equivalent levels of $a$-CT/CTI 
Fig. 3 CTIs negatively regulate fatty acid synthesis through htACCase. a 4-week old plants grown in long-day (LD) conditions. b Above-ground biomass of 4-week-old plants. Data are means \pm SEM, $n=8$ individual biological replicates. Asterisks indicate significant difference from the wild-type control as determined by a two-tailed Student's $t$-test at ${ }^{\star \star \star} P<0.001$. c htACCase activity in the soluble and pellet fractions of crude chloroplasts from 4-week-old rosette leaves. Data are means \pm SEM $n=3$ individual biological replicates. Asterisks indicate a significant difference from the wild-type control as determined by a two-tailed Student's $t$-test at ${ }^{\star \star \star} P<0.001$. $\mathbf{d}$ Rates of fatty acid synthesis in detached rosettes leaves estimated thanks to the incorporation of labeled acetate. Data are means \pm SEM,$n=3$ biologically independent plants. Asterisks indicate significant difference from the wild-type control as determined by a two-tailed Student's t-test at ${ }^{* \star} P<0.001,{ }^{\star \star} P<0.01$, and ${ }^{\star} P<0.05$, respectively. e Leaf total fatty acid content determined by gas chromatography. Data are means \pm SEM,$n=6$ individual biological replicates. Asterisks indicate significant difference from the wild-type control as determined by a two-tailed Student's $t$-test at ${ }^{\star \star}{ }^{\star} P<0.001$, and ${ }^{\star} P<0.05$, respectively. $\mathbf{f}$ Desaturation ratio among C18 fatty acids in rosette leaves. Data are means \pm SEM, scatterplots show individual biological replicates as circles. Asterisks indicate significant difference from the wild-type control as determined by a two-tailed Student's $t$-test at ${ }^{\star \star \star} P<0.001$. $\mathbf{g}$ Accumulation of triacylglycerols (TAG) in rosette leaves of $c t i$ mutants observed by thin-layer chromatography. Experiments were repeated three times with similar results. $\mathbf{h}$ Triacylglycerol (TAG) composition of rosette leaves as determined by ESI-tandem mass spectrometry. The numbers between brackets indicate the number of carbon atoms of the TAG species analyzed. Data are means \pm SEM, $n=5$ biologically independent plants. Asterisks indicate significant difference from the wild-type control as determined by a two-tailed Student's $t$-test at ${ }^{\star \star \star} P<0.001,{ }^{\star \star} P<0.01$, and ${ }^{\star} P<0.05$, respectively.

interactions in all the leaves, regardless of the light/dark regime previously imposed (Fig. 5d, e and Supplementary Fig 14). Lightdependent variations in CTI1/a-CT interactions detected in splitluciferase assays were not due to differential protein accumulation (Supplementary Fig. 14a). To further demonstrate the lightdependence of CTI1/a-CT interaction, co-immunoprecipitation experiments were carried out using leaf samples harvested after different light/dark treatments. In agreement with split-luciferase assays, an extended $(24 \mathrm{~h})$ dark period reduced the interaction between $\alpha$-CT and CTI (Fig. 5f). A strong interaction between the proteins could be restored by an extended $16 \mathrm{~h}$ light illumination. These observations suggest that light-dependent CTIs/a-CT interactions play an important role in the regulation of htACCase and FAS. In summary, we identified a membrane regulatory subunit to the plant htACCase, and light appears to trigger both positive ${ }^{5,6}$ and negative regulatory mechanisms targeting FAS. This is evident from light-induced CTI/a-CT interactions attenuating htACCase activity and limiting carbon flux into de novo FAS (Fig. 5g). We suggest this permits the plant cell to finely regulate the allocation of products of photosynthesis, thus optimizing carbon and energy homeostasis in response to changing environmental and developmental parameters.

\section{Discussion}

De novo fatty acid synthesis in higher plants occurs mainly in plastids, and is catalyzed by a series of enzymes and acyl-carrier proteins $^{33}$. For thermodynamic efficiency, it was proposed that the enzymes, substrates and cofactors for FAS may be compartmentalized to form a metabolic channel ${ }^{36}$. However, we lack a clear picture of such a FAS metabolon. Another dilemma regards how does de novo FAS in the plastids 'communicate' with the cytosol to meet the acyl chain demand of the entire cell? One possibility would be that FAS enzymes sense cellular demand across the envelope by directly associating with this membrane conduit $^{20}$. The carboxylation of acetyl-CoA to malonyl-CoA by ACCase is the first committed step of FAS, and is considered ratelimiting for $\mathrm{FAS}^{37}$. ACCase was detectable on the inner envelope membrane of pea plastids ${ }^{38}$. Subsequently, a protein of $96 \mathrm{kDa}$ (IEP96) associated with the chloroplast inner envelope membrane was characterized ${ }^{18,19}$. IEP96, which shares high similarity with the $35 \mathrm{kDa}$ a-carboxyltransferase subunit of ACCase from Escherichia coli, proved to be the a-CT subunit of plant htACCase ${ }^{18,19}$. The CT subcomplex of htACCase is tightly membrane associated whereas BC/BCCP subcomplex can be partially dissociated from chloroplast membranes ${ }^{20}$. Because of the strong association of $\alpha$-CT with the chloroplast envelope $e^{19,20}$ and the lack of a predicted transmembrane domain in $a-C T$, it was proposed that a chloroplast membrane protein may mediate such membrane association of $a-\mathrm{CT}^{20}$. In this study, we used the noncatalytic domain of $\alpha$-CT as bait in a yeast two-hybrid screen, and we identified an uncharacterized gene, that we termed CTI1. A survey of the Arabidopsis genome revealed two genes related to CTI1, CTI2, and CTI3 (Supplementary Fig.2). All three CTI proteins localize to the plastid envelope membrane and interact specifically with the noncatalytic domain of $\alpha$-CT (Figs. 1,2). The thorough characterization of $c t i$ mutants generated by CRISPR/ Cas9 technology showed that CTI1 is the predominant isoform with respect to the molecular function of the CTIs (Fig. 3). This could be attributed to (i) a higher expression level of CTI1 (Supplementary Fig. 11) combined with (ii) a higher affinity of CTI1 to $\alpha$-CT (Fig. 1c). In agreement with these observations, CTI1 appears to have coevolved with htACCase. The occurrence of CTI2/CTI3 in graminaceous monocots (Supplementary Fig. 2), which lack htACCase, then raises the unresolved question of the function of these homologs in such species.

While FAS in leaves is essential to support the production of membrane lipids and waxes, overproduction of FAs can lead to membrane damage so that excess FAs are detoxified via TAG biosynthesis and accumulation, or via degradation through betaoxidation pathway ${ }^{39}$. TAGs function as a buffer for cytotoxic FAs and other lipid intermediates, thereby playing a key role to maintain intracellular lipid homeostasis 39,40 . Leaf TAG production is energetically wasteful though ${ }^{39,40}$. Under normal circumstances, FAS is highly regulated in vegetative tissues to minimize TAG production ${ }^{40}$. The $a$-CT/CTI module clearly participates in this regulatory mechanism: illumination promotes $\alpha$-CT/CTI interactions, which in-turn attenuates htACCase activity, thus reducing carbon flux into FAS (Fig. 5). Decreased C18 fatty acid desaturation ratio was observed in both in leaves and seeds of $c t i$ mutants. This phenotype probably originates from the disconnection between the FAS module in the plastid and FA modification/elongation modules in the endoplasmic reticulum, the location of production of polyunsaturated fatty acids (PUFAs) and very long chain fatty acids (VLCFAs). FA modifications seem to function at the same rate regardless of de novo FA production capacity. In seeds of Arabidopsis $p I I$ mutants $^{16}$ and transgenic rapeseed lines overexpressing $A C C 1$ in plastids ${ }^{41}$, increased ACCase activity was associated with decreased proportion of polyunsaturated FAs, just like in cti mutants. Reciprocally, in wri1 mutant seeds, FA production is decreased, and the relative proportions of PUFAs and VLCFAs are higher ${ }^{16,29}$.

Light exposure upregulates FAS by activating htACCase $e^{4,5,37}$. Illumination first creates optimal physiological conditions for htACCase by raising $\mathrm{pH}$ and concentrations of $\mathrm{Mg}^{2+}, \mathrm{ATP}$, and reductants. Light-regulated post-translational modifications of catalytic subunits of htACCase have also been described ${ }^{42}$. More 
a

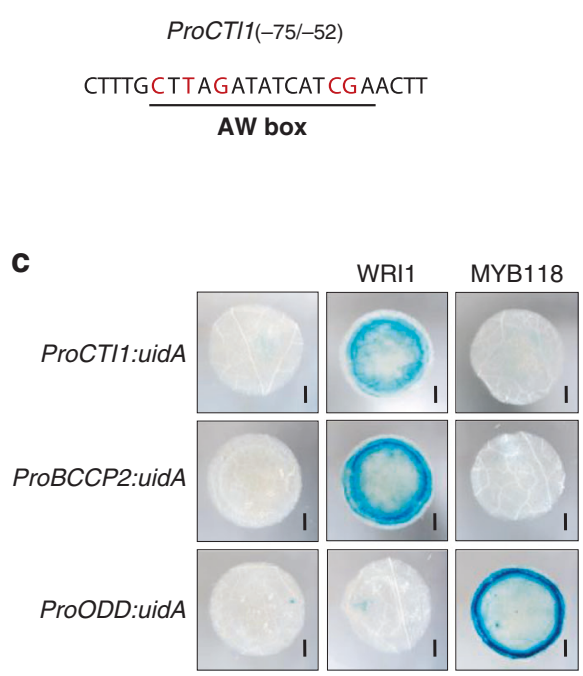

e

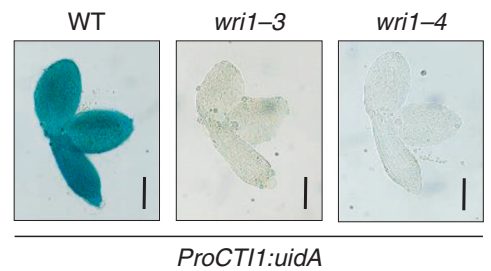

b

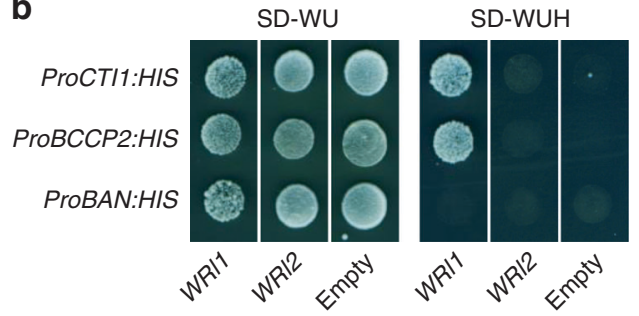

d
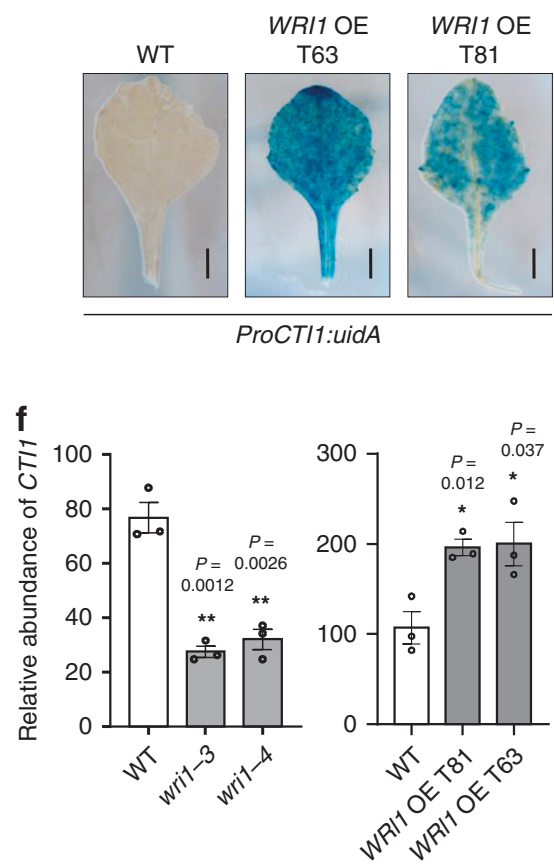

Fig. 4 WRINKLED1 enhances CTI1 expression through direct binding to the CTI1 promoter. a AW cis-regulatory element present in the CTI1 promoter. Conserved nucleotides denoting the presence of an AW box are in red. The numbers between brackets indicate the position of the element relative to the start codon. b WRI1 directly activates the CTI1 promoter in yeast one-hybrid assay. Yeast strains containing the HIS3 reporter gene under the control of either the BANYULS (BAN, negative control), BCCP2 (positive control), or CT/1 promoter were transformed with either the empty expression vector or with versions of the vectors allowing expression of WRI1 or WRI2 (negative control) before being plated on synthetic dropout (SD-WU or SD-WUH) media. The experiment was repeated five times (with independent clones) with similar results. c WRI1 activates the promoter of CTI1 in transactivation assay in leaves of Nicotiana benthamiana. Pro:uidA reporter constructs (ProBCCP2 was used as positive control, ProODD as negative control) alone or in combination with a vector allowing the expression of WRI1 or MYB118 (negative control) were coinfiltrated in young leaves of $\mathrm{N}$. benthamiana. The experiment was repeated twice with similar results. For each experiment and for each condition tested, six independent infiltrations were realized. Representative pictures are displayed. Bars $=2 \mathrm{~mm}$. d Activity of the ProCTI1(994 bp):uidA cassette in rosette leaves of Pro35Sdual:WR1 over-expressing (OE) lines (WRI1 OE T63 and T81) and in a wild-type (WT) background. The experiment was repeated twice (using independent ProCT/1(994 bp):uidA transformants) with similar results. For each experiment and for each genotype, six different leaves were observed. Representative pictures are displayed. Bars $=5 \mathrm{~mm}$. e Activity of the ProCTI1(994 bp):uidA cassette in wri1 mutant embryos and in a WT embryo. The experiment was repeated twice (using independent ProCTI1(994 bp): uidA transformants) with similar results. For each experiment and for each genotype, 10 different embryos were observed. Representative pictures are displayed. Bars $=150 \mu \mathrm{m}$. f Accumulation levels of CTI1 transcripts in seeds of wri1 and in leaves of two independent WRI1 overexpressing lines (WRI1 OE T81 and T63) analyzed by qRT-PCR. The results obtained are standardized to the constitutive EF7 $\alpha A 4$ gene expression level. Data are means \pm SEM, $n=3$ biologically independent plants. Asterisks indicate significant difference from the wild-type control as determined by a two-tailed Student's $t$-test at ${ }^{\star \star} P<0.01$ and ${ }^{\star} P<0.05$, respectively.

recently, $\mathrm{pH}$ modulation of $\mathrm{BADC} / \mathrm{BCCP}$ association within the biotin carboxylase subcomplex was identified and proposed to contribute to light-dependent regulation of the activity of htACCase ${ }^{11}$. BADC subunits were shown to inhibit ACCase in the dark through dark-favored interactions with $\mathrm{BC}$, which correlates with lower htACCase activity and FAS in the dark ${ }^{11}$. To study the effects of light on CTI-mediated FAS regulation, we carried out ${ }^{14} \mathrm{C}$-acetate labeling assays to quantify the FAS rates. ${ }^{14} \mathrm{C}$-acetate labeling in FAS is light-dependent, and has been proposed to be achieved by light-dependent carboxylation of acetyl-CoA by htACCase ${ }^{31-33}$. Like other studies, we found that
${ }^{14} \mathrm{C}$-acetate labeling is a very practical approach to study htACCase in vivo ${ }^{6,7,33,43}$. It revealed that CTIs attenuate the rate of FAS in the light. In addition, ${ }^{14} \mathrm{C}$-acetate labeling suggests that both the accumulation of fatty acids in leaves of cti mutants and $\mathrm{CTI} / \alpha-\mathrm{CT}$ interactions are regulated by light (Fig. 5). Altogether, these data demonstrate that interactions of CTI isoforms with the a-CT subunits regulate FAS in a light-dependent manner.

Phosphorylation of a-CT was previously described and two phosphorylation sites (Ser741 and Ser744 on Arabidopsis ortho$\log$ ) were identified by phosphoproteomic studies ${ }^{44,45}$. Both of the identified a-CT phosphorylation sites are near the 
a
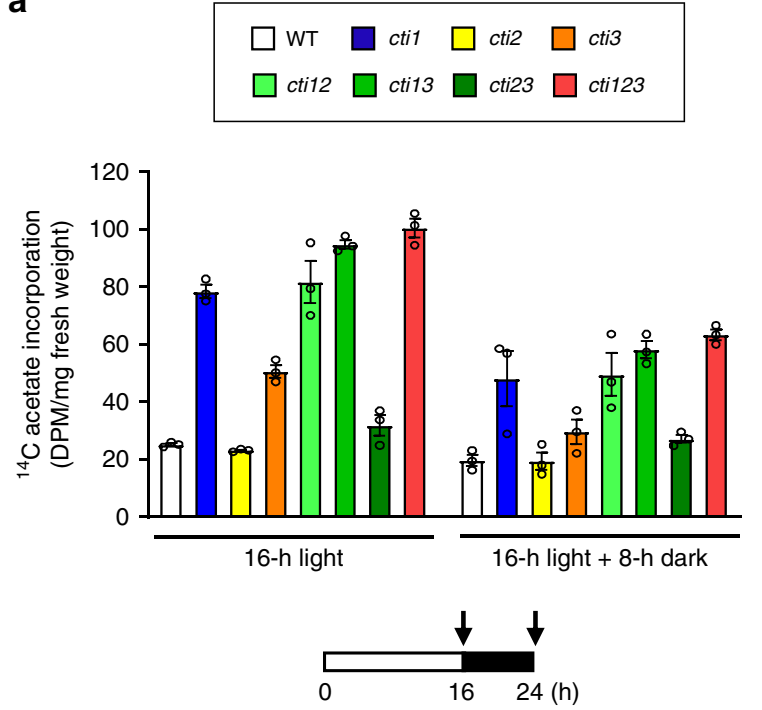

b
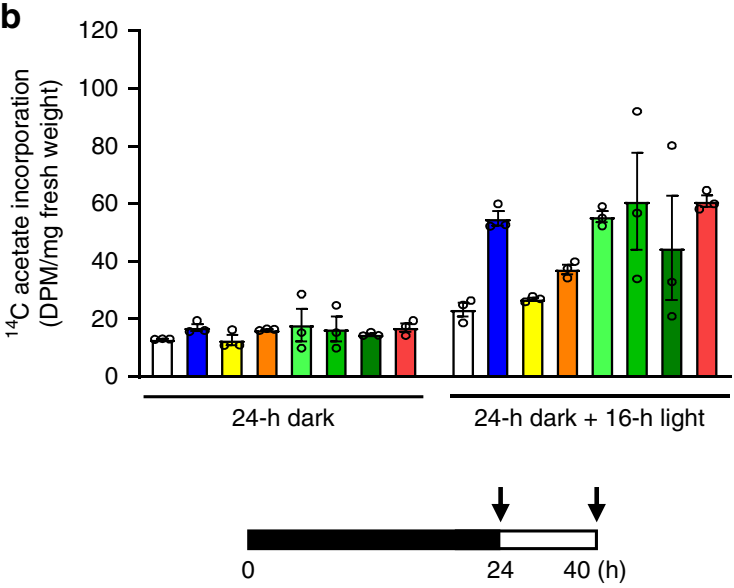

C

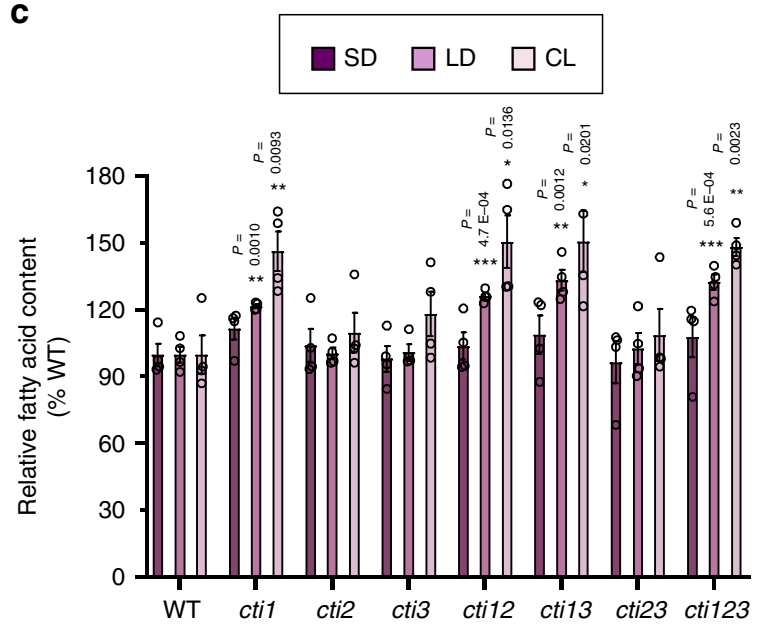

d

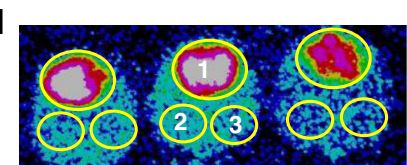

$\begin{array}{lll}\text { 4-d light } & \text { 3-d light } & 2 \text {-d light } \\ & \text { 1-d dark } & 2-d \text { dark }\end{array}$

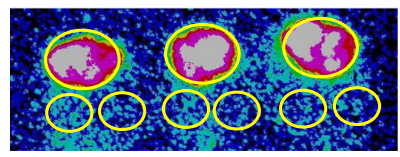

+1 -d light (recovery)

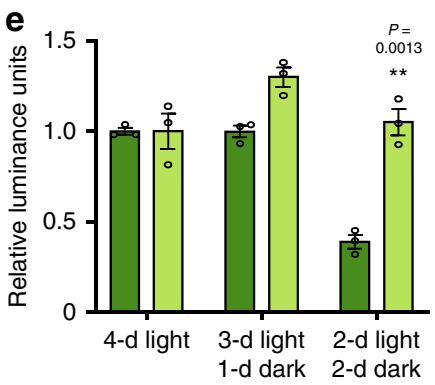

f
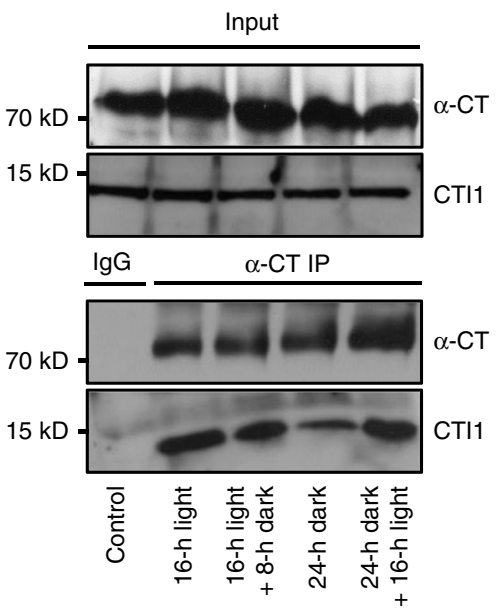

9

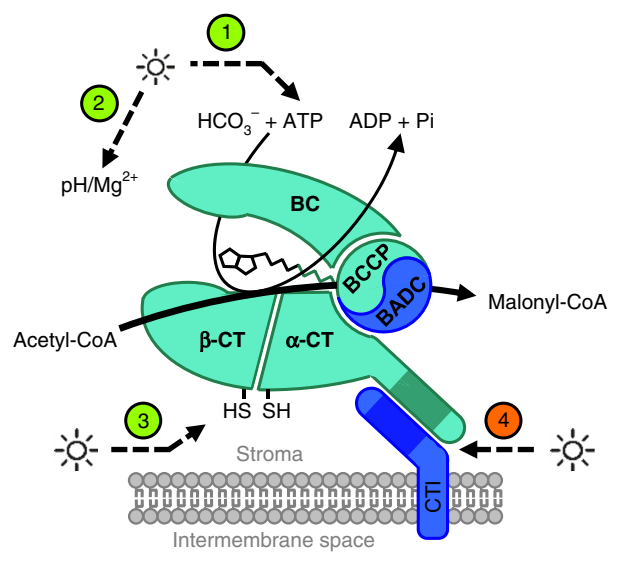

C-terminus, within the noncatalytic domain of the protein, which is responsible for its interaction with CTI. Additionally, a different phosphoproteomic study identified phosphorylation sites on the the C-terminus of CTI1 (Ser160, Thr161) and CTI3 $\left(\right.$ Thr151, Ser152, and Ser153) ${ }^{46}$. The identified phosphorylation sites are conserved in all three CTIs. Protein phosphorylation can be used to modulate the nature and the strength of protein- protein interactions thereby regulating protein binding 47 . Considering the occurrence of phosphorylation sites in the protein binding regions (coiled-coil domains) of both a-CT and CTIs, it would be interesting to further study whether such protein modifications affect the dynamics of CTI/a-CT binding.

Vegetative tissues in ctil mutants display measurable TAG accumulation, resulting in at least a four-fold increase in 
Fig. 5 CTIs interacting with $\boldsymbol{\alpha}$-CT regulate fatty acid synthesis in a light-dependent manner. $\mathbf{a}, \mathbf{b}{ }^{14} \mathbf{C}$-acetate incorporation experiments carried out on rosette leaves aged 4 weeks and subjected to different light/dark regimes. Data are means $\pm S E M, n=3$ biologically independent plants. $\mathbf{c}$ Leaf total fatty acid content of rosette leaves from plants grown under different light regimes as determined by gas chromatography. Plants were grown in short-day (SD), long-day (LD) or constant light (CL) conditions for 4 weeks before analyses. Data are means $\pm S E M, n=4$ biologically independent plants. Asterisks indicate significant difference from the wild-type control as determined by a two-tailed Student's $t$-test at ${ }^{\star \star \star} P<0.001,{ }^{\star \star} P<0.01$, and ${ }^{\star} P<0.05$, respectively. $\mathbf{d}$ Split-luciferase assays. Three combinations of constructs were injected in leaves of Nicotiana benthamiana. After transformation, the plants were grown under different light/dark regimes before imaging of luminescence. Experiments were repeated three times with similar results. For each of the three independent transformations, three different leaves were observed. e Relative luminescence signal quantified after coexpression of CTI1-nLUC and $\alpha$ $\mathrm{CT}$-cLUC as displayed in $\mathbf{d}$. Data are means $\pm \mathrm{SEM}, n=3$ biologically independent plants. Asterisks indicate significant difference from the 2 - $d$ light/2ddark treatment (before recovery) as determined by a two-tailed Student's $t$-test at ${ }^{\star \star} P<0.01$. $\mathbf{f}$ Co-immunoprecipitation assays. IgG was used as a negative control. Experiments were repeated three times with similar results. $\mathbf{g}$ Model for the regulation of htACCase activity by light. Light upregulates FAS by increasing the ATP/ADP ratio (1), optimizing $\mathrm{Mg}^{2+}$ concentration and $\mathrm{pH}$ (2), and redox potential in the plastid (3). Light also attenuates htACCase activity by enhancing $\alpha-C T / C T I$ interactions, possibly on a different timescale (4). Additionally, BADC proteins modulate htACCase activity by competing with BCCP subunits for access to BC.

extractable leaf oil compared with wild-type Arabidopsis leaves (Fig. 3h). From a biotechnological point of view, CTIs may thus represent promising targets for the engineering of vegetative oil crops. While oilseed-based fuel production can meet only a small proportion of world transportation needs before impinging on the global food supply, the overproduction of oil in non-seed tissues such as leaves and stems may be exploited as part of an overall strategy to maximize the recovery of renewable resources from agricultural products ${ }^{39,48}$.

\section{Methods}

Plant material and growth conditions. Arabidopsis thaliana accession Col-0 was used as wild-type control in this study. The T-DNA mutant line (cti1-2; N599125) was ordered from ABRC (Arabidopsis Biological Resource Center). Characterization of the wril-3 and wril-4 T-DNA insertion lines and construction of the Pro35Sdual:WRI1 lines (T63 and T81) were previously reported ${ }^{28}$. All plants were grown in growth chamber at $22^{\circ} \mathrm{C}$. Light intensity was $82-115$ (98 on average) $\mu \mathrm{mol} \mathrm{m} \mathrm{m}^{-2} \mathrm{~s}^{-1}$ and humidity was $50 \%$. The long-day (LD) photoperiod corresponded to an alternate $16 \mathrm{~h}$ light $/ 8 \mathrm{~h}$ dark regime. The short-day (SD) photoperiod corresponded to an alternate $12 \mathrm{~h}$ light $/ 12 \mathrm{~h}$ dark regime.

\section{Constructs preparation and stable transformation of Arabidopsis. The primers} used for construct preparation are listed in Supplementary Table 3.

We designed single-guide RNAs (sgRNA) for CRISPR/Cas9 using the online CRISPR-P 2.0 software $^{49}$. We annealed the oligo pairs to generate double-stranded DNA and cloned the sgRNAs into the BbsI site of the gateway compatible entry vector U6-sgRNA. We then introduced the U6-sgRNA cassette into a modified pCambia1300 binary vector, in which $h s p$ Cas 9 expression is driven by the YAO promoter $^{50}$

To construct ProCTI1:CTI1:uidA, ProCTI2:CTI2:uidA, and ProCTI3:CTI3:uidA transgenes, gene fragments extending from up to $2 \mathrm{~kb}$ upstream of the ATG of the gene to the last coding codon were amplified with the Pfu Ultra DNA polymerase (Stratagene) from Col-0 genomic DNA. The PCR products were introduced by BP recombination into the pDONR207 entry vector (Invitrogen) and transferred into the destination vector pBI101-R1R2-GUS ${ }^{51}$ by LR recombination. The resulting binary vector was electroporated into Agrobacterium tumefaciens C58C strain and used for transformation ${ }^{51}$. Depending on the construct considered, between 17 and 19 independent transgenic lines were analyzed. To construct the ProCTI1:uidA transgene, region -994 to -1 bp relative to the CTI1 translational start codon was introduced by BP recombination into the pDONR207 entry vector as described above, and transferred into the destination vector pBI101-R1R2-GUS by LR recombination. Construction of the ProBCCP2:uidA transgene ${ }^{28}$, just like that of the ProODD:uidA transgene ${ }^{52}$ followed a similar procedure.

To construct ProAT2S2:CTI transgenes, CTI cDNAs were amplified from a mixture of seed cDNA (Col-0 accession), cloned into the pDONR207 as described above, and finally transferred to the binary vector ProAT2S2-R1R2-HYGRO ${ }^{53}$ by LR recombination. The corresponding binary vectors were used for agroinfiltration of cti1-2 flower buds. T2 seeds were subjected to segregation analyses for hygromycin resistance and lines segregating 3:1 were selected (heterozygous lines, one insertion locus). T2 lines were then grown in a greenhouse and their progeny (T3 seeds) was subjected to segregation analyses. Lines producing $100 \%$ resistant plantlets were selected (homozygous lines, single insertion locus) and used for further characterization.

To construct Pro35S:CTI1:YFP, Pro35S:CTI2:YFP, Pro35S:CTI3:YFP, and Pro35S: $\alpha-C T: Y F P$ transgenes for protoplast transient transformation, CTI and $\alpha-$ $C T$ coding sequences (CDSs) without STOP codons were amplified from a mixture of cDNA (Col-0 accession), cloned into the pENTR/D-TOPO, and transferred into the pAM-PAT-35SS::YFP:GW vector ${ }^{54}$ by LR recombination.

To construct Pro35S:CTI1:GFP, Pro35S: $\alpha-C T: R F P$, and Pro35S:TGD2:RFP transgenes, CDSs were cloned into the $\mathrm{pENTR/D-TOPO}$ vector as above, before being transferred into destination vectors pGWB605 (GFP) and pGWB654 (RFP) by $\mathrm{LR}$ recombination ${ }^{55}$.

To construct BiFC and split-luciferase vectors, CDSs previously cloned into the pENTR/D-TOPO were transferred into pAM-PAT-35SS::cYFP/nYFP:GW, and pEarley-nLUC/cLUC vectors by LR recombination.

To purify the coiled-coil domains of the CTIs and to perform yeast two-hybrid assays, the CDSs corresponding to CTI coiled-coil domains were amplified by PCR cloned into the pENTR/D-TOPO entry vector and transferred into destination vectors pGADT7-GW by LR recombination.

To purify the proteins for microscale thermophoresis, the C-terminus of CTI1, CTI3, and $\alpha$-CT were each fused with a HIS tag, and cloned into pET30a (CTI3 and $\alpha-\mathrm{CT}$ ) or pDEST17 (CTI1). CTI2 was fused with a GST tag and cloned into the pGEX4T-1 vector.

To construct the Pro35S:CTI1:HA transgene, a HindIII/StuI fragment of the pGWB14 binary vector ${ }^{55}$ containing a $35 \mathrm{~S}$ promoter-R1-CmR-ccdB-R2-3xHANosT sequence was HindIII/SmaI inserted in the pBluescript KS(+) vector (Stratagene), giving the $\mathrm{pBKS}-35 \mathrm{~S}-\mathrm{R} 1 \mathrm{R} 2-3 \mathrm{xHA}$ vector. CTI1 CDS without STOP codon previously cloned into the pDONR207 (see above) was transferred into the pBKS-35S-R1R2-3xHA vector by LR recombination. To construct the ProCTI1: CTI1:HA transgene, the CTI1 gene was amplified from Col-0 genomic DNA and the $3 \mathrm{xHA}$ tag was amplified from the pBKS-35S-R1R2-3xHA containing the Pro35S:CTI1:HA fusion (see above). The two amplified fragments were combined together to serve as overlapping templates for a PCR reaction utilizing primers listed in Supplementary Table 3. The final PCR product was cloned in pDONR207, and finally transferred into the binary vector pBIB-Hyg-GTW ${ }^{51}$ by LR recombination. The corresponding binary vector was used for agroinfiltration of cti1-2. Homozygous lines (single insertion locus) were selected as described above.

To construct the Pro35S:TOC33:MYC transgene, the CDS corresponding to TOC33 was amplified by PCR, cloned into the pENTR/D-TOPO entry vector and transferred into destination vectors pGWB617 by LR recombination.

To construct the Pro35Sdual:WRI1 ${ }^{51}$ and Pro35Sdual:MYB118 transgenes $^{52}$, the corresponding CDSs were amplified by PCR, cloned into the pDONR207 entry vector by $\mathrm{BP}$ recombination and transferred into the pMDC32 destination by LR recombination

RNA isolation and quantitative real-time PCR. For RNA extraction, frozen tissues were ground in liquid nitrogen and total RNA was extracted using the RNeasy Plant Mini Kit (Qiagen) according to the manufacturer's instructions. For reverse transcription, RNA was converted into first-strand cDNA using the SuperScript preamplification system for first-strand cDNA synthesis (ThermoFisher) with oligo $(\mathrm{dT})_{22}$. The real-time quantitative RT-PCR reaction was performed on the LightCycler Instrument (Roche) with the LightCycler-FastStar DNA Master SYBR Green I kit for PCR (Roche) according to the manufacturer's protocol. Each reaction was performed with $5 \mu \mathrm{L}$ of $1: 50(\mathrm{v} / \mathrm{v})$ dilution of the first cDNA strands in a total volume of $20 \mu \mathrm{L}$. The reaction was incubated at $95{ }^{\circ} \mathrm{C}$ for $8 \mathrm{~min}$ to activate the hot start recombinant Taq DNA polymerase, followed by 45 cycles of $10 \mathrm{~s}$ at 95 ${ }^{\circ} \mathrm{C}, 6 \mathrm{~s}$ at $55^{\circ} \mathrm{C}$, and $20 \mathrm{~s}$ at $72^{\circ} \mathrm{C}^{56}$. Specific primer sequences are presented in Supplementary Tables 4,5 . The specificity of the PCR amplification was checked with a heat dissociation protocol (from 65 to $95^{\circ} \mathrm{C}$ ) following the last cycle of the PCR.

Yeast one-hybrid assay. Construction of the ProCTI1-500:HIS reporter plasmid, like that of the ProBCCP2-180:HIS reporter plasmid ${ }^{29}$, relied on the amplification by PCR of promoter fragments, that were digested by EcoRI and SacII, and inserted into the pHISi vector between the EcoRI and SacII sites. The plasmids thus 
obtained were digested with $\mathrm{NcoI}$ and integrated into the yeast strain YM4271 at the URA3 locus. Yeast cells presenting the HIS3 reporter gene under the control of the different promoters studied were transformed with pDEST22 using LiAc/ SSDNA/PEG method. Yeast cells were grown in YPDA medium and harvested by centrifugation at $3000 \times g$. For each transformation, around $10^{8}$ cells were mixed with T Mix (240 $\mu \mathrm{L}$ PEG $335050 \%(\mathrm{w} / \mathrm{v}), 36 \mu \mathrm{L} 1 \mathrm{M}$ lithium acetate, $50 \mu \mathrm{L}$ boiled SS-Carrier DNA ( $2 \mathrm{mg} / \mathrm{mL}), 34 \mu \mathrm{L}$ plasmid DNA (0.1-1 $\mu \mathrm{g})$ ). After vigorous mix by vortex, the mixtures were incubated in a $28^{\circ} \mathrm{C}$ water bath for $30 \mathrm{~min}$, then in a $42^{\circ} \mathrm{C}$ water bath for $25 \mathrm{~min}$. Afterwards, cells were pelleted by centrifugation, washed with sterile water, resuspended in $200 \mu \mathrm{L}$ sterile water before being plated on selective medium and grown at $30^{\circ} \mathrm{C}$ for $3 \mathrm{~d}$.

Yeast two-hybrid assays. The cDNA library used was prepared by introducing Arabidopsis cDNA prepared from seedlings into the pGATD7-GW vector. The screening assay was conducted according to the Clontech yeast handbook. Briefly, the sequence encoding the coiled-coil domain of a-CT (amino acid 420-769) was cloned into the pGBKT7 vector. This vector was used to transform the AH109 yeast strain. Yeast transformants were selected on synthetic dropout (SD) medium lacking tryptophan. Then, $100 \mu \mathrm{g}$ of cDNA library were used to transform AH109 cells containing the pGBKT7 vector expressing the coiled-coil domain of $\alpha$-CT. Cells plated on SD medium lacking leucine, tryptophan, histidine, adenine, were incubated at $30^{\circ} \mathrm{C}$ for $4 \mathrm{~d}$ and cDNAs of positive clones were amplified by PCR and sequenced using T7 and 3'AD primers (Supplementary Table 6).

For oriented interaction assays, the sequences coding for the coiled-coil domain of CTIs were cloned into the pGADT7 vector as described above. Then, the AH109 strain was cotransformed with the pGADT7 vector expressing CTI coiledcoil domains and the pGBKT7 vector expressing the $\alpha$-CT coiled-coil domain before being plated on SD medium lacking leucine and tryptophan. After 3 days at $30^{\circ} \mathrm{C}$, positive clones were transferred to $\mathrm{SD}$ medium lacking leucine, histidine, and tryptophan, in the presence of $1 \mathrm{mM} 3 \mathrm{AT}$ for another $4 \mathrm{~d}$ at $30^{\circ} \mathrm{C}$.

Transformation of Arabidopsis protoplasts. Arabidopsis protoplasts were prepared from 4-week-old Col-0 plants ${ }^{57}$. About 40 leaves were cut into strips with a sharp razor blade and then transferred into $20 \mathrm{~mL}$ enzyme solution $(20 \mathrm{mM}$ MES $\mathrm{pH} 5.7,1.5 \%(\mathrm{w} / \mathrm{v})$ cellulase R10, 0.4\% (w/v) macerozyme R10, $0.4 \mathrm{M}$ mannitol, $20 \mathrm{mM} \mathrm{KCl}$ and $0.1 \% \mathrm{BSA}$ ) for $3 \mathrm{~h}$ at room temperature. After $3 \mathrm{~h}$, the enzyme solution was filtered through $75-\mu \mathrm{m}$ nylon mesh that was then washed with $20 \mathrm{~mL}$ W5 solution ( $2 \mathrm{mM}$ MES pH 5.7, $154 \mathrm{mM} \mathrm{NaCl}, 125 \mathrm{mM} \mathrm{CaCl}_{2}, 5 \mathrm{mM} \mathrm{KCl}$ ). The flow-through was centrifuged at $200 \times g$ for $2 \mathrm{~min}$. After removing as much supernatant as possible, the protoplast pellet was resuspended in $1 \mathrm{~mL} \mathrm{W5}$ solution and kept on ice for $30 \mathrm{~min}$. The protoplasts were pelleted at $200 \times \mathrm{g}$ for $2 \mathrm{~min}$ and resuspended in $500 \mu \mathrm{L}$ MMG solution (4 mM MES pH 5.7, $0.4 \mathrm{M}$ mannitol, $15 \mathrm{mM}$ $\left.\mathrm{MgCl}_{2}\right)$. Then, $10 \mu \mathrm{L}$ purified plasmid $(10-20 \mu \mathrm{g})$ were mixed with $100 \mu \mathrm{L}$ protoplasts before $110 \mu \mathrm{L}$ PEG solution, namely $40 \%$ (w/v) PEG4000 in $\mathrm{ddH}_{2} \mathrm{O}$ containing $0.2 \mathrm{M}$ mannitol and $100 \mathrm{mM} \mathrm{CaCl}$, were added. The transfection reaction was mixed by gently tapping the tube and incubated at room temperature for 10 $\mathrm{min}$. The mixture was then diluted in $500 \mu \mathrm{L}$ W5 solution, mixed by gently rocking the tube to stop the transfection process, and ultimately centrifuged for $2 \mathrm{~min}$ at $200 \times g$. The pellet was resuspended in $1 \mathrm{~mL} \mathrm{W5}$ solution and incubated overnight at $23^{\circ} \mathrm{C}$ under weak light.

\section{Transient expression in leaves of Nicotiana benthamiana. Agrobacterium} tumefaciens strains transformed with the different binary vectors prepared were grown overnight in selective medium. Cells were then pelleted, washed two times and resuspended in injection buffer (50 mM MES pH 5.7, $10 \mathrm{mM} \mathrm{MgCl}_{2}$ ). Different strain combinations were coinfiltrated into the leaves of Nicotiana benthamiana in the presence of a vector coding for the P19 protein of tomato bushy stunt virus (TBSV) that prevents the onset of post-transcriptional gene silencing.

Confocal microscopy. Fluorescence was observed by confocal laser microscopy using a Leica TCS SP8 device. Fluorescence were observed at $488-505 \mathrm{~nm}$ for GFP, 514-527 $\mathrm{nm}$ for YFP, and $558-583 \mathrm{~nm}$ for RFP.

Split-luciferase assay. After agrobacterium-mediated infiltration of N. benthamiana leaves, different light/dark treatments were applied and luciferase activity was detected with a CCD camera by applying firefly D-luciferin (Goldbio). For 24$\mathrm{h}$ light recovery assays, after luminescence imaging, the detached leaves were kept in a tray with water to maintain humidity. After $24 \mathrm{~h}$ of light recovery, the luciferase activity was detected with the CCD camera again. Ponceau S Staining ( $1 \%$ (w/v) Ponceau S, 20\% acetic acid) was used to quantify relative protein contents.

Microscale thermophoresis (MST). The coiled-coil domains of CTI1, CTI3, and $a-C T$ fused with HIS tag were purified by capture on nickel agarose resin. The coiled-coil domain of CTI2 fused with a GST tag was purified by capture with glutathione resin. Each protein was expressed in Luria Broth. Expression was induced with $100 \mu \mathrm{M}$ IPTG when $\mathrm{OD}_{600}$ reached 0.6 . The cells were pelleted and resuspended with lysis buffer (PBS buffer $(137 \mathrm{mM} \mathrm{NaCl}, 2.7 \mathrm{mM} \mathrm{KCl}, 10 \mathrm{mM}$ $\mathrm{Na}_{2} \mathrm{HPO}_{4}$ and $2 \mathrm{mM} \mathrm{KH_{2 }} \mathrm{PO}_{4}$, $\mathrm{pH}$ 7.4). After cell disruption by French press and centrifugation, the proteins were purified by nickel agarose resin (Gold Biotechnology, H-320) or glutathione agarose resin (Gold Biotechnology, G-250). The purified protein samples were concentrated to $500 \mu \mathrm{L}$ with a Pierce concentrator PES with a 3000 Dalton cutoff (ThermoFisher, 88527). The GST tag was proteolytically separated from CTI2 using thrombin treatment. Proteins were quantified by Bradford assays using bovine gamma globulin as a standard. $100 \mathrm{nM}$ a-CT-His protein was fluorescently labeled using His-tag labeling kit (NanoTemper, MOL008) according to the user manual. After labeling, $10 \mu \mathrm{L}$ of labeled $\alpha$-CT protein was mixed with $10 \mu \mathrm{L}$ of CTI (serial dilutions in PBS buffer). The microthermophoresis was carried out using 100\% LED power and high MST power with a NanoTemper monolith NT.115 instrument. The data were analyzed by MO. Offinity Analysis (X86).

Immunoblotting. For quantification of the htACCase subunit content, proteins were extracted from crude chloroplasts using a protein extraction buffer $(50 \mathrm{mM}$ HEPES-KOH pH 7.6, $150 \mathrm{mM} \mathrm{NaCl}, 1 \mathrm{mM}$ EDTA, 10\% (v/v) glycerol, $1 \mathrm{mM} \mathrm{2-}$ mercaptoethanol, $1 \%(\mathrm{v} / \mathrm{v})$ Triton X-100, 1X complete protease inhibitor cocktail). The crude chloroplasts were isolated from $5 \mathrm{~g}$ of 4 -week-old Col-0 leaf tissues. The proteins were separated by SDS-PAGE and blotted to a PVDF membrane. The BCCP, $\alpha$-CT and $\beta$-CT subunits of plastidic htACCase were immunologically detected with dedicated antisera ${ }^{19}$. A horseradish peroxidase-conjugated secondary antibody was used and the horseradish peroxidase activity was detected by enhanced chemiluminescence (ECL) western blotting substrate (ThermoFisher; Cat. No. 32106). Ponceau S Staining was used as a loading control.

Co-immunoprecipitation. Crude chloroplasts were isolated from 4-week-old Col-0 plants. Around $5 \mathrm{~g}$ of fresh leaves were ground in $30 \mathrm{~mL}$ ice-cold isolation buffer (50 mM HEPES pH 8.0, $2 \mathrm{mM}$ EDTA, $2.5 \mathrm{mM} \mathrm{MgCl}_{2}, 5 \mathrm{mM} \mathrm{NaHCO}_{3}, 0.33 \mathrm{M}$ sorbitol, $0.5 \% \mathrm{BSA})$. After filtration of the homogenate through miracloth, the flow-through was centrifuged at $1000 \times g$ for $10 \mathrm{~min}$ at $4{ }^{\circ} \mathrm{C}$. The pellet was resuspended in $1 \mathrm{~mL}$ of protein extraction buffer containing $50 \mathrm{mM}$ Tris $\mathrm{pH} 7.5$, $150 \mathrm{mM} \mathrm{NaCl}, 1 \%$ Triton-X 100 and $1 \mathrm{x}$ protease inhibitor cocktail (Sigma). After a 30-min incubation period on ice, the solution was centrifuged at $20,000 \times g$ for 15 min at $4{ }^{\circ} \mathrm{C}$. The supernatant was decanted before addition of $5 \mu \mathrm{L}$ of anti- $\alpha$-CT antibody and subsequent incubation for $4 \mathrm{~h}$ with end-to-end rotation at $4{ }^{\circ} \mathrm{C}$. After addition of $25 \mu \mathrm{L}$ of protein A resin (Genescript; Cat. No. L00210), another $2 \mathrm{~h}$ incubation period was implemented. The mixture was finally spun down and washed three times with washing buffer $(50 \mathrm{mM}$ Tris $\mathrm{pH} 7.5,150 \mathrm{mM} \mathrm{NaCl})$. After washing, the resin was incubated with $100 \mu \mathrm{L}$ of $1 \times$ SDS-PAGE loading buffer, and then heated at $80^{\circ} \mathrm{C}$ for $10 \mathrm{~min}$. Proteins were separated by SDS-PAGE and then transferred to PVDF membrane. After transfer, the proteins were detected by immunoblotting with anti-CTI1 or anti- $\alpha$-CT antibody. A horseradish peroxidaseconjugated secondary antibody was used and the horseradish peroxidase activity was detected by ECL western blotting substrate (ThermoFisher; Cat. No. 32106).

Protease protection assay. Four-week-old leaves of transgenic plants stably expressing a TOC33-MYC fusion were harvested and $10 \mathrm{~g}$ of fresh material were used to isolate crude chloroplasts according to method described above. Chloroplasts were resuspended in $1 \mathrm{ml}$ of isolation buffer and loaded onto a Percoll gradient. The Percoll gradient was obtained by mixing $15 \mathrm{~mL}$ of Percoll with $15 \mathrm{~mL}$ of $2 \times$ isolation buffer, and centrifuging the mixture at $38,700 \times g$ for $30 \mathrm{~min}$ at $4{ }^{\circ} \mathrm{C}$ The chloroplasts were centrifuged on the Percoll gradient using a prechilled swinging bucket rotor at $7700 \times g$ for $10 \mathrm{~min}$ at $4{ }^{\circ} \mathrm{C}$ with no brake. After centrifugation, the upper green band corresponding to broken chloroplasts was removed and discarded. The lower green band was transferred into a new $50-\mathrm{mL}$ centrifuge tube containing $10 \mathrm{~mL}$ of isolation buffer. The mixture was spun down at $1500 \times g$ for $5 \mathrm{~min}$ at $4^{\circ} \mathrm{C}$, and the pellet resuspended in $1 \mathrm{~mL}$ reaction buffer (50 mM HEPES pH 8.0, 0.33 M sorbitol). The proteolytic digestions were set up as follows: mock $-150 \mu \mathrm{L}$ of chloroplasts were mixed with $100 \mu \mathrm{L}$ of reaction buffer thermolysin $-150 \mu \mathrm{L}$ of chloroplasts were mixed with $10 \mu \mathrm{L}$ of thermolysin stock solution $\left(1 \mathrm{mg} / \mathrm{mL}\right.$, freshly prepared in $5 \mathrm{mM} \mathrm{CaCl}_{2}$ in reaction buffer) and $90 \mu \mathrm{L}$ of reaction buffer; trypsin- $150 \mu \mathrm{L}$ of chloroplasts were mixed with $10 \mu \mathrm{L}$ of trypsin stock solution $(1 \mathrm{mg} / \mathrm{mL}$, freshly made in reaction buffer) and $90 \mu \mathrm{L}$ of reaction buffer. All reactions were incubated on ice for $30 \mathrm{~min}$. Each proteolytic reaction was quenched on ice for $5 \mathrm{~min}$ as follows: mock $-50 \mu \mathrm{L}$ of reaction buffer was added; thermolysin $-50 \mu \mathrm{L}$ of quench solution (60 mM EDTA in reaction buffer) was added; trypsin- $50 \mu \mathrm{L}$ of trypsin inhibitor solution $(1 \mathrm{mg} / \mathrm{mL}$ in reaction buffer; Sigma; Cat. No. T6522) was added. SDS-PAGE loading buffer was then added to each reaction. Western blotting was performed as described above using anti-Myc, anti- $\alpha-C T$ or anti-CTI1 antibodies.

Immunolocalization experiments. Coverslips were sterilized in $95 \%$ ethanol and dried before coating. They were then incubated for $5 \mathrm{~min}$ at $37^{\circ} \mathrm{C}$ in a $50 \mu \mathrm{g} / \mathrm{mL}$ poly-L-lysine solution before drying. Arabidopsis protoplasts were fixed for $30 \mathrm{~min}$ with $3 \%(\mathrm{w} / \mathrm{v})$ paraformaldehyde in PBS supplemented with $30.5 \mathrm{~g} / \mathrm{L}$ glucose and $30.5 \mathrm{~g} / \mathrm{L}$ mannitol (pH 7.4; PBS-GM) at room temperature. Fixed protoplasts were washed three times with $50 \mathrm{mM}$ ammonium chloride in PBS-GM. The final suspension was plated onto poly-L-lysine-coated coverslips. Protoplasts were allowed to settle for $10 \mathrm{~min}$ at room temperature, and were then air dried for $1 \mathrm{~h}$ under 
culture hood. To permeabilize protoplasts, a 15 min treatment with $0.5 \%(\mathrm{v} / \mathrm{v})$ Triton X-100 buffered with PBS-GM was carried out. After three washes with PBSGM, nonspecific binding was blocked with $1 \%$ bovine serum albumin in PBS-GM for $1 \mathrm{~h}$. Protoplasts were then incubated overnight at $4{ }^{\circ} \mathrm{C}$ with rabbit anti-E37 antibodies ${ }^{19}$ (1:500) followed by $1 \mathrm{~h}$ incubation at room temperature with rat antiHA antibodies (1:2000; Roche). Cells were washed for $20 \mathrm{~min}$ in PBS-GM, and incubated with goat anti-rabbit immunoglobulin G-Alexa Fluor 568 (1:1000; Molecular Probes) and with goat anti-rat immunoglobulin G-Alexa Fluor 488 (1:500; Molecular Probes) or with goat anti-mouse immunoglobulin G-Alexa Fluor 488 (1:500; Molecular Probes) for $1 \mathrm{~h}$. Finally, cells were washed for $20 \mathrm{~min}$ in PBS and mounted using Vestashield mounting solution (Vector Laboratories). Cells were visualized with an inverted spectral confocal laser microscope (LEICA SP2AOBS). Preparation of embryos excised from maturing seeds was similar to that of protoplasts except for the permeabilization treatment: cell walls were partially digested by incubating embryos in 2\% (w/v) driselase (Sigma) in PBS-GM for 45 min, then membranes were permeabilized by a $1 \mathrm{~h}$ treatment with $10 \%(\mathrm{v} / \mathrm{v})$ DMSO and 3\% (v/v) NP-40 in PBS-GM.

Lipid and fatty acid analyses. To analyze polar lipids and triacylglycerols, $500 \mathrm{mg}$ of leaves were harvested and stored at $-80^{\circ} \mathrm{C}$ prior to lipid extraction. Leaf samples were ground in $7.2 \mathrm{~mL}$ of precooled chloroform/methanol/formic acid $(10: 10: 0.5, \mathrm{v} / \mathrm{v} / \mathrm{v})$ and incubated at $-20^{\circ} \mathrm{C}$ overnight. The mixture was centrifuged at $7500 \times g$ for $10 \mathrm{~min}$ at $4{ }^{\circ} \mathrm{C}$ to pellet the cell debris. Lipids of the pellet were reextracted in $2.64 \mathrm{ml}$ of precooled chloroform/methanol/water $(10: 10: 1, \mathrm{v} / \mathrm{v} / \mathrm{v})$. Then, $3.6 \mathrm{~mL}$ of cooled Hajra solution $\left(2 \mathrm{M} \mathrm{KCl}\right.$ and $\left.0.2 \mathrm{M} \mathrm{H}_{3} \mathrm{PO}_{4}\right)$ were added to the pooled supernatants. After shaking and centrifugation $(7500 \times g$ for $10 \mathrm{~min}$ at $4{ }^{\circ} \mathrm{C}$ ), the lower phase containing lipids was collected and evaporated with a stream of $\mathrm{N}_{2}$. Lipids were resuspended in chloroform/methanol (2:1, v/v) and separated on thin-layer chromatography plates developed with hexane/diethylether/acetic acid $(35: 15: 0.01, \mathrm{v} / \mathrm{v} / \mathrm{v})$. Polar lipids and triacylglycerols were visualized under UV light by staining with sprayed primuline. Lipid spots were ultimately collected and analyzed by gas chromatography ${ }^{58}$.

To analyze total fatty acids in leaves or seeds, total lipids were transmethylated into fatty acid methyl esters (FAMEs). FAMEs were analyzed by a Hewlett Packard 6890 gas chromatograph. Leaves from 4-week-old plants were collected and dried by centrivap SpeedVac overnight. Mature seeds were harvested and dried at room temperature for 1 week prior to analysis.

Lipidomics. Lipid extracts were dissolved in chloroform, and appropriate amounts of internal standards were introduced. The lipids were detected by triple quadrupole mass spectrometer (Applied Biosystems API 4000) with an autosampler (LC Mini PAL; CTC Analytics ${ }^{59}$. Data processing was performed in a Lipidome DB Data Calculation Environment (http://lipidome.bcf.ku.edu:9000/Lipidomics) with normalization to internal standards.

In vivo acetate labeling. In vivo labeling experiments were performed according to previous report ${ }^{60}$. Leaves from 4 -week-old plants were cut into strips after measuring the fresh weight. The leaf strips were transferred into $2 \mathrm{~mL}$ of reaction buffer $(20 \mathrm{mM}$ MES pH 5.5, half MS, $0.01 \%(\mathrm{v} / \mathrm{v})$ Tween 20$)$ in a 6-well plate. The labeling assays were started by the addition of $1 \mu \mathrm{Ci}$ of ${ }^{14} \mathrm{C}$-acetate (PerkinElmer; Cat. No. NEC084H001MC) or $1 \mu \mathrm{Ci}$ of ${ }^{14} \mathrm{C}$-acetate (Morevek; Cat. No. MC213). After this, the 6-well plates were incubated on a shaker in the light $\left(40 \mu \mathrm{mol} \mathrm{m}^{-2} \mathrm{~s}^{-1}\right)$ at room temperature or in the dark. The samples were collected after $40 \mathrm{~min}$ of incubation, washed three times with water and total lipids were then extracted in $800 \mu \mathrm{L}$ of methanol/chloroform/formic acid (20:10:1, v/v/v) with vortexing for 10 seconds $^{61}$. The mixture was incubated for $30 \mathrm{~min}$ at $22^{\circ} \mathrm{C}$, complemented with $500 \mu \mathrm{L}$ of $1 \mathrm{M}$ $\mathrm{KCl}$ and $0.2 \mathrm{M} \mathrm{H}_{3} \mathrm{PO}_{4}$, vortexed and centrifuged at $12,000 \times g$ for $30 \mathrm{sec}$ for collection of the chloroform phase containing the lipids. One milliliter of scintillation cocktail was added to this phase before measurement of the incorporated radioactivity (in $\mathrm{cpm})$ with a scintillation counter.

ACCase activity assay. ACCase activity was directly quantified from 4-week-old leaves by incorporation of $\mathrm{H}^{14} \mathrm{CO}_{3}$ into acid-stable products. Around $5 \mathrm{~g}$ of leaf tissues were harvested. Crude chloroplasts were isolated as above, and chloroplast proteins were extracted using $300 \mu \mathrm{L}$ of extraction buffer $(50 \mathrm{mM}$ Tris- $\mathrm{HCl} \mathrm{pH} \mathrm{7.5,}$ $100 \mathrm{mM} \mathrm{KCl}, 5 \mathrm{mM} \mathrm{MgCl} 2,1 \mathrm{mM} \mathrm{DTT}, 1 \%$ (v/v) Triton X-100, 10\% (v/v) glycerol, and $1 \times$ plant protease inhibitor mixture from Sigma). After centrifugation at $13,000 \times g$ for $10 \mathrm{~min}$, the supernatants were desalted with spin desalting columns (ThermoFisher; Cat. No. 89882). The insoluble pellets were resuspended in the extraction buffer. Reactions were initiated by mixing $10 \mu \mathrm{L}$ of reaction buffer (100 mM Tricine $\mathrm{pH} 8.2,100 \mathrm{mM} \mathrm{KCl}, 15 \mathrm{mM}$ ATP, $5 \mathrm{mM} \mathrm{MgCl}, 1 \mathrm{mM}$ DTT, $2.5 \mathrm{mM}$ acetyl-CoA, $50 \mu \mathrm{M}$ haloxyfop, and $1 \mu \mathrm{Ci}{ }^{14} \mathrm{C}-\mathrm{NaHCO}_{3}$ (Moravek; Cat. No. MC208) with $40 \mu \mathrm{L}$ of protein extract. After $30 \mathrm{~min}$ at RT, the reactions were quenched by adding $50 \mu \mathrm{L}$ of $12 \mathrm{~N} \mathrm{HCl}$. The reaction solutions were transferred into scintillation vials with a filter paper at the bottom of the vial. Samples were dried at $80^{\circ} \mathrm{C}$ for $1 \mathrm{~h}$, after which $1 \mathrm{~mL}$ of scintillation cocktail was added and radioactivity counted by liquid scintillation. Controls without acetyl-CoA were included to determine and subtract nonspecific background rates.
GUS assay. For histochemical detection of GUS activity, tissues were incubated in $0.1 \mathrm{M}$ phosphate buffer, $\mathrm{pH} 7.2$ containing $2 \mathrm{mM}$ 5-bromo-3-indolyl- $\beta$-D-glucuronide, $0.1 \%(\mathrm{v} / \mathrm{v})$ Triton X-100, $10 \mathrm{mM} \mathrm{Na} \mathrm{N}_{2}$-EDTA, and 0.2 (Arabidopsis vegetative organs, flowers, and seeds) or $2 \mathrm{mM}$ (Arabidopsis embryos and $N$. benthamiana leaf disks) each of potassium ferricyanide and potassium ferrocyanide. A vacuum was applied for $1 \mathrm{~h}$ before incubating the samples for $4 \mathrm{~h}$ (Arabidopsis embryos and $N$. benthamiana leaf disks) or $15 \mathrm{~h}$ (Arabidopsis vegetative organs, flowers, and seeds) at $37^{\circ} \mathrm{C}$ in the dark, and chlorophyll was finally removed by room temperature incubation in $70 \%(\mathrm{v} / \mathrm{v})$ ethanol (vegetative tissues and flowers only).

Reporting summary. Further information on research design is available in the Nature Research Reporting Summary linked to this article.

\section{Data availability}

The authors declare that the data supporting the findings of this study are available within the article and its Supplementary Information files. The datasets and plant materials generated and analyzed during the current study are available from the corresponding author upon reasonable request. Sequence data from this article can be found in TAIR under accession numbers: AT2S2, At4g27150; BAN, At1g61720: BC, At5g35360; BCCP1, At5g16390; BCCP2, At5g15530; CTI1, At1g42960; CTI2, At3g02900 CTI3, At5g16660; $\alpha$-CT, At2g38040; $\beta$-CT, AtCg00500; EF1 $\alpha A 4$, At5g60390; MYB118, At3g27785; ODD, Atlg04380; PTST2, Atlg27070; TGD2, At3g20320; TOC33, At1g02280; WRI1, At3g54320; WRI2, At2g41710. Source data are provided with this paper.

Received: 29 April 2020; Accepted: 9 November 2020; Published online: 03 December 2020

\section{References}

1. Thelen, J. J. \& Ohlorogge, J. B. Metabolic engineering of fatty acid biosynthesis in plants. Metab. Eng. J. 4, 12-21 (2002).

2. Kleinig, H. \& Liedvogel, B. On the energy requirements of fatty acid synthesis in spinach chloroplasts in the light and in the dark. FEBS Lett. 101, 2-5 (1979).

3. Sauer, A. \& Heise, K. P. On the light dependence of fatty acid synthesis in spinach chloroplasts. Plant Physiol. 73, 11-15 (1983).

4. Sasaki, Y., Kozaki, A. \& Hatano, M. Link between light and fatty acid synthesis: thioredoxin-linked reductive activation of plastidic acetyl-CoA carboxylase. Proc. Natl Acad. Sci. USA 94, 11096-11101 (1997).

5. Kozaki, A. \& Sasaki, Y. Light-dependent changes in redox status of the plastidic acetyl-CoA carboxylase and its regulatory component. Biochem. J. 339, 541-546 (1999).

6. Andre, C., Haslam, R. P. \& Shanklin, J. Feedback regulation of plastidic acetylCoA carboxylase by 18:1-acyl carrier protein in Brassica napus. Proc. Natl Acad. Sci. USA 109, 10107-10112 (2012).

7. Bates, P. D. et al. Fatty acid synthesis is inhibited by inefficient utilization of unusual fatty acids for glycerolipid assembly. Proc. Natl Acad. Sci. USA 111, 1204-1209 (2014).

8. Bourrellier, A. B. F. et al. Chloroplast acetyl-CoA carboxylase activity is 2oxoglutarate-regulated by interaction of PII with the biotin carboxyl carrier subunit. Proc. Natl Acad. Sci. USA 107, 502-507 (2010).

9. Salie, M. J., Zhang, N., Lancikova, V., Xu, D. \& Thelen, J. J. A family of negative regulators targets the committed step of de novo fatty acid biosynthesis. Plant Cell 28, 2312-2325 (2016).

10. Shivaiah, K. K., Ding, G., Upton, B. \& Nikolau, B. J. Non-catalytic subunits facilitate quaternary organization of plastidic acetyl-CoA carboxylase. Plant Physiol. 182, 756-775 (2020).

11. Ye, Y. et al. The BADC and BCCP subunits of chloroplast acetyl-CoA carboxylase sense the $\mathrm{pH}$ changes of the light-dark cycle. J. Biol. Chem. 295, 9901-9916 (2020).

12. Mason, J. M. \& Arndt, K. M. Coiled coil domains: stability, specificity, and biological implications. ChemBioChem. 5, 170-176 (2004).

13. Awai, K., Xu, C., Tamot, B. \& Benning, C. A phosphatidic acid-binding protein of the chloroplast inner envelope membrane involved in lipid trafficking. Proc. Natl Acad. Sci. USA 103, 10817-10822 (2006).

14. Seung, D. et al. Two plastidial coiled-coil proteins are essential for normal starch granule initiation in Arabidopsis. Plant Cell 30, 1523-1542 (2018).

15. Ferro, M. et al. AT-CHLORO, a comprehensive chloroplast proteome database with subplastidial localization and curated information on envelope proteins. Mol. Cell. Proteomics 9, 1063-1084 (2010).

16. Baud, S. et al. PII is induced by WRINKLED1 and fine-tunes fatty acid composition in seeds of Arabidopsis thaliana. Plant J. 64, 291-303 (2010).

17. Breuers, F., et al. Dynamic remodeling of the plastid envelope membranes-a toll for chloroplast envelope in vivo localizations. Front. Plant Sci. https://doi. org/10.3389/fpls.2012.00007 (2012). 
18. Hirsch, S. \& Soll, J. Import of a new chloroplast inner envelope protein is greatly stimulated by potassium phosphate. Plant Mol. Biol. 27, 1173-1181 (1995).

19. Shorrosh, B. S., Savage, L. J., Soll, J. \& Ohlrogge, J. B. The pea chloroplast membrane-associated protein, IEP96, is a subunit of acetyl-CoA carboxylase. Plant J. 10, 261-268 (1996).

20. Thelen, J. J. \& Ohlrogge, J. B. The multisubunit acetyl-CoA carboxylase is strongly associated with the chloroplast envelope through non-ionic interactions to the carboxyltransferase subunits. Arch. Biochem. Biophys. 400, 245-257 (2002).

21. Teyssier, E., Block, M., Douce, R. \& Joyard, J. Is E37, a majorpolypeptide of the inner membrane from plastid envelope, an S-adenosyl methionindependentmethyltransferase? Plant J. 10, 903-912 (1996).

22. Motohashi et al. Functional analysis of the $37 \mathrm{kDa}$ inner envelope membrane polypeptide in chloroplast biogenesis during Ds-tagged Arabidopsis palegreen mutant. Plant J. 34, 719-731 (2003).

23. Cheng, Z. et al. Highly divergent methyltransferases catalyze a conserved reaction in tocopherol and plastoquinone synthesis in Cyanobacteria and photosynthetic eukaryotes. Plant Cell 15, 2343-2356 (2003).

24. Xiang, S. et al. A different mechanism for the inhibition of the carboxyltransferase domain of acetyl-coenzyme A carboxylase by tepraloxydim. Proc. Natl Acad. Sci. USA 106, 20723-20727 (2009).

25. Pouvreau, B. et al. Duplicate maize wrinkled1 transcription factors activate target genes involved in seed oil biosynthesis. Plant Physiol. 156, 674-686 (2011).

26. Cernac, A. \& Benning, C. WRINKLED1 encodes an AP2/EREB domain protein involved in the control of storage compound biosynthesis in Arabidopsis. Plant J. 40, 575-585 (2004).

27. Maeo, K. et al. An AP2-type transcription factor, WRINKLED1, of Arabidopsis thaliana binds to the AW-box sequence conserved among proximal upstream regions of genes involved in fatty acid synthesis. Plant J. 1, 476-487 (2009).

28. Baud, S. et al. Function of plastidial pyruvate kinases in seeds of Arabidopsis thaliana. Plant J. 52, 405-419 (2007).

29. Baud, S., Wuillème, S., To, A., Rochat, C. \& Lepiniec, L. Role of WRINKLED1 in the transcriptional regulation of glycolytic and fatty acid biosynthetic genes in Arabidopsis. Plant J. 60, 933-947 (2009).

30. Liu, H. et al. WRINKLED1 regulates BIOTIN ATTACHMENT DOMAINCONTAINING proteins that inhibit fatty acid synthesis. Plant Physiol. 181, 55-62 (2019)

31. Nakamura, Y. \& Yamada, M. The light-dependent step of de novo synthesis of long chain fatty acids in spinach chloroplasts. Plant Sci. Lett. 14, 291-295 (1979).

32. Roughan, G., Kagawa, T. \& Beevers, H. On the light dependency of fatty acid synthesis by isolated spinach chloroplasts. Plant Sci. Lett. 18, 221-228 (1980).

33. Post-Beittenmiller, D., Roughan, G. \& Ohlrogge, J. B. Regulation of plant fatty acid biosynthesis. Plant Physiol. 100, 923-930 (1992).

34. Ke, J. et al. The role of pyruvate dehydrogenase and acetyl-coenzyme A synthetase in fatty acid synthesis in developing Arabidopsis seeds. Plant Physiol. 123, 497-508 (2000).

35. Bao, X., Focke, M., Pollard, M. \& Ohlrogge, J. Understanding in vivo carbon precursor supply for fatty acid synthesis in leaf tissue. Plant J. 22, 39-50 (2000).

36. Roughan, P. \& Ohlrogge, J. B. Evidence that isolated chloroplasts contain an integrated lipid-synthesizing assembly that channels acetate into long-chain fatty acids. Plant Physiol. 110, 1239-1247 (1996).

37. Hunter, S. C. \& Ohlrogge, J. B. Regulation of spinach chloroplast acetyl-CoA carboxylase. Arch. Biochem. Biophys. 359, 170-178 (1998).

38. Sasaki, Y. et al. Chloroplast-encoded protein as a subunit of acetyl-CoA carboxylase in pea plant. J. Biol. Chem. 268, 25118-25123 (1993).

39. Xu, C. \& Shanklin, J. Triacylglycerol metabolism, function, and accumulation in Plant vegetative tissues. Annu. Rev. Plant Biol. 67, 179-206 (2016).

40. Chapman, K. D. \& Ohlrogge, J. B. Compartmentation of triacylglycerol accumulation in plants. J. Biol. Chem. 287, 2288-2294 (2012).

41. Roesler, K., Shintani, D., Savage, L., Boddupalli, S. \& Ohlrogge, J. Targeting of the Arabidopsis homomeric acetyl-coenzyme A carboxylase to plastids of rapeseeds. Plant Physiol. 113, 75-81 (1997).

42. Savage, L. \& Ohlrogge, J. Phosphorylation of pea chloroplast acety-CoA carboxylase. Plant J. 18, 521-527 (1999).

43. Keereetaweep, J., Liu, H., Zhai, Z. \& Shanklin, J. Biotin attachment domaincontaining proteins irreversibly inhibit acetyl CoA Carboxylase. Plant Physiol. 177, 208-215 (2018).

44. Meyer, J. L., Gao, J., Xu, D. \& Thelen, J. J. Phosphoproteomic analysis of seed maturation in Arabidopsis, rapeseed and soybean. Plant Physiol. 159, 517-528 (2012).

45. Agrawal, G. K. \& Thelen, J. J. Large scale identification and quantitative profiling of phosphoproteins expressed during seed filling in oilseed rape. Mol. Cell. Proteomics 5, 2044-2059 (2006).
46. Umezawa, T. et al. Genetics and phosphoproteomics reveal a protein phosphorylation network in the abscisic acid signaling pathway in Arabidopsis thaliana. Sci. Signal. 6, re8 (2013).

47. Nishi, H., Hashimoto, K. \& Panchenko, R.,A. Phosphorylation in protein protein binding: effect on stability and function. Structure 19, 1807-1815 (2011).

48. James, C. N. et al. Disruption of the Arabidopsis CGI-58 homologue produces Chanarin -Dorfman-like lipid droplet accumulation in plants. Proc. Natl Acad. Sci. USA 107, 17833-17838 (2010).

49. Liu, H. et al. CRISPR-P 2.0: an improved CRISPR-Cas9 tool for genome editing in plants. Mol. Plant. 20, 530-530 (2017).

50. Yan, L. et al. High-efficiency genome editing in Arabidopsis using YAO promoter-driven CRISPR/Cas9 system. Mol. Plant 8, 1820-1823 (2015).

51. To, A. et al. WRINKLED transcription factors orchestrate tissue-specific regulation of fatty acid biosynthesis in Arabidopsis. Plant Cell 24, 5007-5023 (2013).

52. Barthole, G. et al. MYB118 represses endosperm maturation in seeds of Arabidopsis. Plant Cell 26, 3519-3537 (2014)

53. Pouvreau, B. et al. Duplicate maize wrinkled1 transcription factors activate target genes involved in seed oil biosynthesis. Plant Physiol. 156, 674-686 (2011).

54. Chen, D. et al. Extracellular ATP elicits DORN1-mediated RBOHD phosphorylation to regulate stomatal aperture. Nat. Commun. 8, 2265-2278 (2017).

55. Nakagawa, T. et al. Development of series of gateway binary vectors, pGWBs, for realizing efficient construction of fusion genes for plant transformation. $J$. Biosci. Bioeng. 104, 34-41 (2007).

56. Baud, S., Vaultier, M. \& Rochat, C. Structure and expression profile of the sucrose synthase multigene family in Arabidopsis. J. Exp. Bot. 55, 397-409 (2004).

57. Yoo, S. D., Cho, Y. H. \& Sheen, J. Arabidopsis mesophyll protoplasts: a versatile cell system for transient gene expression analysis. Nat. Protoc. 2, 1565-1572 (2007).

58. Baud, S. et al. An integrated overview of seed development in Arabidopsis thaliana ecotype WS. Plant Physiol. Biochem. 40, 151-160 (2002).

59. Chen, M. \& Thelen, J. J. ACYL-LIPID DESATURASE2 is required for chilling and freezing tolerance in Arabidopsis. Plant Cell 25, 1430-1444 (2013).

60. Fan, J., Zhai, Z., Yan, C. \& Xu, C. Arabidopsis TRIGALACTOSYLDIACYLGLYCEROL5 interacts with TGD1, TGD2, and TGD4 to facilitate lipid transfer from the endoplasmic reticulum to plastids. Plant Cell 27, 2941-2955 (2015).

61. Dormann, P., Hoffmann-Benning, S., Balbo, I. \& Benning, C. Isolation and characterization of an Arabidopsis mutant deficient in the thylakoid lipid digalactosyl diacylglycerol. Plant Cell 7, 1801-1810 (1995).

\section{Acknowledgements}

We thank N. Nesi and J. Jouhet for helpful discussion and the Kansas State University Lipidomics Research Center for lipidomics analysis. This research was funded by National Science Foundation (grants MCB-1716688 and PGRP IOS-1829365 to J.J.T.), the Agence Nationale de la Recherche (grant ANR-11-BTBR-0004 to S.B.), and the IJPB benefits from the support of Saclay Plant Sciences-SPS (ANR-17-EUR-0007 to S.B.).

\section{Author contributions}

Y.Y., J.J.T., and S.B. designed the study. Y.Y., K.N., A.T., and E.T.F. performed the experiments. Y.Y. drafted the manuscript, and L.L., E.F., S.R.V., S.B., and J.J.T. revised the manuscript.

\section{Competing interests}

Y.Y. and J.T. are co-inventors on a pending patent pertaining to this study. This patent has been licensed to Yield10 Biosciences, LLC (Woburn, MA) for which the co-inventors receive royalties. All other authors declare no competing interests.

\section{Additional information}

Supplementary information is available for this paper at https://doi.org/10.1038/s41467 020-20014-5.

Correspondence and requests for materials should be addressed to J.J.T.

Peer review information Nature Communications thanks the anonymous reviewers for their contribution to the peer review of this work. Peer review reports are available.

Reprints and permission information is available at http://www.nature.com/reprints

Publisher's note Springer Nature remains neutral with regard to jurisdictional claims in published maps and institutional affiliations. 
(c) (i) Open Access This article is licensed under a Creative Commons Attribution 4.0 International License, which permits use, sharing, adaptation, distribution and reproduction in any medium or format, as long as you give appropriate credit to the original author(s) and the source, provide a link to the Creative Commons license, and indicate if changes were made. The images or other third party material in this article are included in the article's Creative Commons license, unless indicated otherwise in a credit line to the material. If material is not included in the article's Creative Commons license and your intended use is not permitted by statutory regulation or exceeds the permitted use, you will need to obtain permission directly from the copyright holder. To view a copy of this license, visit http://creativecommons.org/ licenses/by/4.0/.

(C) The Author(s) 2020 